\title{
CONVEXITY ESTIMATES FOR LEVEL SETS OF QUASICONCAVE SOLUTIONS TO FULLY NONLINEAR ELLIPTIC EQUATIONS
}

\author{
PENGFEI GUAN AND LU XU
}

\begin{abstract}
We establish a global geometric lower bound for the second fundamental form of the level surfaces of solutions to $F\left(D^{2} u, D u, u, x\right)=0$ in convex ring domains, in terms of boundary geometry and the structure of the elliptic operator $F$. We also prove a microscopic constant rank theorem, under a general structural condition introduced by Bianchini-Longinetti-Salani in [3] .
\end{abstract}

\section{INTRODUCTION}

Solutions of boundary value problems for elliptic equations often inherit important geometric properties of the domains with the influence of the structures of the corresponding equations. One of these geometric features is the quasiconcavity. A function $u$ is called quasiconcave if its level sets $\{x \mid u(x) \geqslant c\}$ are convex. By the work of Gabriel [8], the Green function of a convex domain is quasiconcave. The same is also true for $p$-harmonic functions in convex ring domains with homogeneous boundary conditions following Lewis [12]. Another example is the quasiconcavity of solutions to the free boundary problem arising in plasma physics in convex domains in the work of Caffarelli-Spruck [6]. The quasiconcavity of solutions to nonlinear equations has been studied extensively in the literature, we refer [8, 12, 5, 10, 11, 6, 9, 7, 13, 3, 14, 2] and references therein. The techniques of quasiconcave envelopes have been refined by Colesanti-Salani [6], and more recently by Bianchini-Longinetti-Salani [3] to prove quasiconcavity of solutions to general degenerate elliptic fully nonlinear equations in the form

$$
F\left(D^{2} u, D u, u, x\right)=0,
$$

in convex ring domain $\Omega=\Omega_{0} \backslash \Omega_{1}$ (i.e. $\Omega_{0} \subset \subset \Omega_{1}$ are convex) with the Dirichlet boundary condition

$$
\left.u\right|_{\partial \Omega_{0}}=0, \quad \text { and }\left.u\right|_{\partial \Omega_{1}}=1 .
$$

The main focus of this paper is on the quantified properties of the quasiconcave solutions of equations of form (1.1). More specifically, we establish a global a priori estimate on the geometric lower bound of the principal curvatures of the level surfaces of these quasiconcave solutions, in terms of boundary geometry and the structure of operator $F$. In addition to

1991 Mathematics Subject Classification. 35J925, 35J65, 35B05.

Key words and phrases. level sets, convexity estimates, fully nonlinear elliptic equations.

Research of the first author was supported in part by NSERC Discovery Grant. Research of the second author was supported in part by NSFC No.10901159 and NSFC No.11071245. 
the geometric interest, this type of estimates may be used via homotopic deformation to obtain the existence of quasiconcave solutions of the corresponding equations. We achieve this macroscopic geometric estimate through a microscopic Constant Rank Theorem for the smallest principal curvatures of the level surfaces for quasiconcave solutions. A simple microscopic convexity principle for level surfaces of solutions of equations in form (1.1) is obtained in Theorem [1.3, under a general structural condition introduced in 3 , to cover a larger class of nonlinear equations. A more refined version for the smallest principal curvatures of the level surfaces is proved in the last section of the paper. The main result, Theorem 1.4, is a consequence of this type of microscopic principle.

Let us introduce some notation. Denote $\mathscr{S}_{n}$ the space of real symmetric $n \times n$ matrices and let $\Upsilon \subset \mathscr{S}_{n}$ be an open set.

Definition 1.1. $\forall \theta \in \mathbb{S}^{n-1}$, denote $\theta^{\perp}$ the linear subspace in $\mathbb{R}^{n}$ which is orthogonal to $\theta$. Define $\mathscr{S}_{n}^{-}(\theta)$ to be the class of $n \times n$ symmetric real matrices which are negative definite on $\theta^{\perp}$. Denote $\mathscr{S}_{n}^{0-}(\theta)$ the subclass of $\mathscr{S}_{n}^{-}(\theta)$ of matrices that have $\theta$ as eigenvector with corresponding null eigenvalue. For any $b \in \mathbb{R}^{n}$ with $t=\langle b, \theta\rangle>0$, define

$$
\mathscr{B}_{\theta}^{-}(\Upsilon)=\left\{\mathbf{B} \in \mathscr{S}_{n+1} \mid \mathbf{B}=\left(\begin{array}{cc}
\widetilde{\mathbf{B}} & b^{T} \\
b & \chi
\end{array}\right) \text { with } \quad \widetilde{\mathbf{B}} \in \mathscr{S}_{n}^{0-}(\theta) \cap \Upsilon, \chi \in \mathbb{R}\right\}
$$

Denote $\mathbf{J}=\left(I_{n} \mid \mathbf{0}\right)$ the $n \times(n+1)$ matrix, where $I_{n}$ is the $n \times n$ identity matrix and $\mathbf{0}$ is the null vector in $\mathbb{R}^{n}$. Suppose $F=F(r, p, u, x)$ is a $C^{2}$ function in $\Upsilon \times \mathbb{R}^{n} \times \mathbb{R} \times \Omega$, $\forall(\theta, u) \in \mathbb{S}^{n-1} \times \mathbb{R}$ fixed, set

$$
\Gamma_{F}=\left\{(\mathbf{B}, x) \in \mathscr{B}_{\theta}^{-}(\Upsilon) \times \Omega: F\left(t^{-1} \mathbf{J B}^{-1} \mathbf{J}^{T}, t^{-1} \theta, u, x\right) \geqslant 0\right\} .
$$

The following was proved in [3].

Theorem 1.2. [Bianchini-Longinetti-Salani] Suppose F is proper, continuous, degenerate elliptic operator which satisfies a viscosity comparison principle. Assume that for each $(\theta, u)$ fixed, the super-level set $\Gamma_{F}$ defined in (1.4) is convex. If $u \in C^{2}(\Omega) \bigcap C(\bar{\Omega})$ with $|\nabla u|>0$ is an admissible classical solution of equation (1.1) satisfying the Dirichlet boundary value (1.2) in convex ring domain $\Omega$, then level set $\{x \in \Omega \mid u(x) \geqslant c\} \cup \Omega_{1}$ is convex for each constant $0 \leqslant c \leqslant 1$.

The class of operators $F$ satisfying conditions in Theorem 1.2 includes Laplace operators, $p$-Laplace operators, the Pucci operator, and the mean curvature type equations of the form

$$
\sum_{i, j=1}^{n} a_{i j}(\nabla u, u, x) u_{i j}=f(\nabla u, u, x) .
$$

A similar result was also proved by Bianchini-Longinetti-Salani in [3] under the assumption that, $\forall \theta, u$ fixed

(1.6) $\Xi_{F}=\left\{(\mathbf{A}, t, x) \in \Upsilon \times(0,+\infty) \times \Omega: F\left(t^{-3} \mathbf{A}, t^{-1} \theta, u, x\right) \geqslant 0\right\} \quad$ is locally convex. 
With this structural condition on $F$, a constant rank theorem was obtained in [2]. The convexity structural condition on $F$ in Theorem 1.2 is weaker than the convexity structural condition on $\Xi_{F}$. In particular, the mean curvature operator (1.5) does not satisfy condition (1.6). Detailed discussion of these conditions as well as examples will be given in section 2 .

To establish a strict convexity estimate on the second fundamental forms of the level surfaces of solutions in Theorem [1.2, we need two assumptions:

$$
\text { ellipticity: } \quad\left(F^{\alpha \beta}\right):=\left(\frac{\partial F}{\partial r_{\alpha \beta}}\left(\nabla^{2} u(x), \nabla u(x), u(x), x\right)\right)>0, \quad \forall x \in \Omega
$$

structural condition: $\quad \forall(\theta, u)$ fixed, the set $\Gamma_{F}$ is locally convex.

Throughout the paper, we assume

$$
|D u(x)| \geqslant d_{0}>0, \forall x \in \Omega,
$$

to ensure that the level-surface $\{x \in \Omega \mid u(x)=c\}$ is smooth for each $c$.

The first result of this paper is a microscopic constant rank theorem.

Theorem 1.3. Suppose $u \in C^{3,1}(\Omega)$ is a solution of (1.1) and $\left(D^{2} u(x), D u(x), u(x)\right) \in$ $\Upsilon \times \mathbb{R}^{n} \times\left(-\gamma_{0}+\delta_{0}, \gamma_{0}+\delta_{0}\right)$ for some $\delta_{0} \in \mathbb{R}$ at $x \in \Omega$. Suppose that $F$ satisfies conditions (1.7) - (1.8) and the level set $\{x \in \Omega \mid u(x) \geqslant c\} \cup \Omega_{1}$ of $u$ is connected and locally convex for all $c \in\left(-\gamma_{0}+\delta_{0}, \gamma_{0}+\delta_{0}\right)$ for some $\gamma_{0}>0$. Then the second fundamental form of level surface $\Sigma^{c}=\{x \in \Omega \mid u(x)=c\}$ has the same constant rank for all $c \in\left(-\gamma_{0}+\delta_{0}, \gamma_{0}+\delta_{0}\right)$.

We now switch our attention to global geometric bounds of the second fundamental forms of level surfaces of $u$. For a function $u$ defined in domain $\Omega$, denote

$$
\Sigma^{c}=\{x \in \bar{\Omega} \mid u(x)=c\}
$$

to be the level surface. For any $x \in \Sigma^{c}$, denote $\kappa_{s}(x)$ the smallest principal curvature of the level surface $\Sigma^{c}$ at $x$. For each $c \in \mathbb{R}$, if $\Sigma^{c} \neq \emptyset$, set

$$
\kappa^{c}=\inf _{x \in \Sigma^{c}} \kappa_{s}(x)
$$

We will strengthen (1.7) to

$$
\text { uniform ellipticity: } \exists \lambda>0,\left(\frac{\partial F}{\partial r_{\alpha \beta}}\left(D^{2} u(x), D u(x), u(x), x\right)\right) \geqslant \lambda\left(\delta_{\alpha \beta}\right), \quad \forall x \in \bar{\Omega} .
$$

Set

$$
\varpi=\max _{\alpha, \beta, \gamma, \eta, i, j} \frac{\sup _{x \in \Omega}\left\{\left|\frac{\partial^{2} F\left(D^{2} u(x), D u(x), u(x), x\right)}{\partial r_{\alpha \beta} \partial r_{\gamma \eta}}\left\|\frac{\partial F\left(D^{2} u(x), D u(x), u(x), x\right)}{\partial r_{i j}}\right\| D u(x)\right|\right\}}{\lambda} .
$$

Theorem 1.4. Suppose $u$ is a classical solution of equation (1.1) with the Dirichlet boundary value (1.2) in convex ring domain $\Omega$. Suppose $F$ satisfies conditions (1.8)-(1.10) at $\left(D^{2} u, D u, u, x\right) \in \Upsilon \times \mathbb{R}^{n} \times[0,1] \times \Omega$. Then

$$
\kappa^{c} \geqslant \min \left\{\kappa^{0} e^{A c}, \kappa^{1} e^{A(c-1)}, \frac{\lambda e^{A(c-1)}}{100 \varpi}\right\}, \quad \forall c \in[0,1]
$$


for some universal constant $A \geqslant 0$ depending only on $\|F\|_{C^{2}}, n, \lambda, d_{0},\|u\|_{C^{3}}$.

It should be pointed out that the convexity estimates carried out in this paper are very sensitive to the structure of the corresponding equation. For equations of the form (1.5) with the Dirichlet boundary condition (1.2), the behavior of $f$ is crucial. For instance, in the case of Laplace equation

$$
\Delta u=f(u),
$$

Theorems $1.3-1.4$ are true when $f \geqslant 0$. In general, Theorem 1.3 does not hold if $f(u)<0$ in equation (1.13), even for $f \equiv-1$.

The rest of the paper is organized as follows. In Section 2, we discuss the structural conditions and prove two key lemmas: Lemma 2.5 and Lemma 2.6. An auxiliary curvature test function is analyzed in Section 3. The proof of Theorem 1.3 - Theorem [1.4 is given in the last section, by establishing a strong maximum principle for the test function considered in Section 3.

\section{STRUCTURAL CONDITIONS}

We recall some notation and results in [3].

Definition 2.1. $\forall \theta \in \mathbb{S}^{n-1}$, denote by $\mathscr{A}_{\theta}^{-}(\Upsilon)$ the following open set in $\mathscr{S}_{n+1}$ :

$$
\mathscr{A}_{\theta}^{-}(\Upsilon)=\left\{\mathbf{A} \in \mathscr{S}_{n+1}: \quad \mathbf{A}=\left(\begin{array}{cc}
\widetilde{\mathbf{A}} & \mu \theta^{T} \\
\mu \theta & 0
\end{array}\right) \text { with } \quad \widetilde{\mathbf{A}} \in \mathscr{S}_{n}^{-}(\theta) \cap \Upsilon, \mu>0\right\}
$$

Properties of $\mathscr{A}_{\theta}^{-}, \mathscr{B}_{\theta}^{-}$and their relationship have been studied in [3]. We list some of them which will be used in this paper.

$\operatorname{det} \mathbf{A} \neq 0$ if $\mathbf{A} \in \mathscr{A}_{\theta}^{-}$, and

$$
\mathscr{B}_{\theta}^{-}(\Upsilon)=\left\{\mathbf{A}^{-1}: \mathbf{A} \in \mathscr{A}_{\theta}^{-}(\Upsilon)\right\} .
$$

If $\mathbf{B}=\mathbf{A}^{-1} \in \mathscr{B}_{\theta}^{-}(\Upsilon)$, then

$$
\widetilde{\mathbf{A}}=\mathbf{J B}^{-1} \mathbf{J}^{T} \quad \text { and } \quad \mu=\frac{1}{t} .
$$

Set

$$
Q=t^{2} \mathbf{J B}^{-1} \mathbf{J}^{T}
$$

where $\mathbf{B} \in \mathscr{B}_{\theta}^{-}(\Upsilon)$ and $t$ defined in Definition 1.1. By symmetry of $\mathbf{B}, t=\langle b, \theta\rangle=$ $\sum_{l=1}^{n} B_{n+1} \theta_{l}$.

In what follows, we will use summation over repeated indices $\alpha, \beta, \gamma, \eta, k, l, m, r, s \in$ $\{1, \ldots, n\}$ and $c, d, e, f \in\{1, \ldots, n+1\}$ unless otherwise indicated.

Lemma 2.2. [lemma 3.11 in [3] $Q$ defined in 2.4] is concave in $\mathbf{B} \in \mathscr{B}_{\theta}^{-}(\Upsilon)$. Furthermore

$$
I:=F^{\alpha \beta} \frac{\partial^{2} Q_{\alpha \beta}}{\partial B_{c d} \partial B_{e f}} X_{c d} X_{e f} \leqslant 0
$$


for any nonnegative definite $n \times n$ matrix $\left(F^{\alpha \beta}\right)$ and any $(n+1) \times(n+1)$ symmetric $\operatorname{matrix}\left(X_{c d}\right)$.

Proof. The concavity of $Q$ has been proved in [3]. For any nonnegative definite $n \times n$ matrix $\left(F^{\alpha \beta}\right)$, there exist $\zeta_{1}, \cdots, \zeta_{n} \in \mathbb{R}^{n}$, such that

$$
\left(F^{\alpha \beta}\right)=\zeta_{1} \zeta_{1}^{T}+\cdots+\zeta_{n} \zeta_{n}^{T}
$$

Therefore, $I \leqslant 0$ follows directly from the concavity of $Q$.

For function $F(r, p, u, x)$, write $F^{\alpha \beta}=\frac{\partial F}{\partial r_{\alpha \beta}}, F^{p_{l}}=\frac{\partial F}{\partial p_{l}}, \cdots$ as derivatives of $F$ with respect to corresponding arguments. For the level set $\Gamma_{F}$ defined in (1.4), denote the tangent space of $\Gamma_{F}$ as

$\mathcal{T} \Gamma_{F}=\left\{V=\left(\left(X_{c d}\right),\left(Z_{k}\right)\right) \in \mathscr{S}_{n+1} \times \mathbb{R}^{n}:\left\langle V, \nabla_{(\mathbf{B}, x)} F\left(t^{-1} \mathbf{J B}^{-1} \mathbf{J}^{T}, t^{-1} \theta, u, x\right)\right\rangle=0\right\}$.

Write

$$
F\left(t^{-1} \mathbf{J B}^{-1} \mathbf{J}^{T}, t^{-1} \theta, u, x\right)=F\left(t^{-3} Q, p, u, x\right) .
$$

Condition (1.8) is equivalent to the fact

$$
V \nabla_{(\mathbf{B}, x)}^{2} F V^{T} \leqslant 0, \quad \forall V=\left(\left(X_{c d}\right),\left(Z_{k}\right)\right) \in \mathcal{T} \Gamma_{F} .
$$

A straight computation yields,

$$
\begin{aligned}
\nabla_{\mathbf{B}} F & =\left(\frac{F^{\alpha \beta}}{t^{4}}\left(t \frac{\partial Q_{\alpha \beta}}{\partial B_{c d}}-3 Q_{\alpha \beta} \delta_{n+1 c} \delta_{l d} \theta_{l}\right)-\frac{F^{p_{s}}}{t^{2}} \delta_{n+1 c} \delta_{l d} \theta_{s} \theta_{l}\right), \\
\nabla_{x} F & =\left(F^{x_{1}}, \ldots, F^{x_{n}}\right),
\end{aligned}
$$

$V \nabla_{(\mathbf{B}, x)}^{2} F V^{T}=\frac{F^{\alpha \beta, \gamma \eta}}{t^{8}}\left(t \frac{\partial Q_{\alpha \beta}}{\partial B_{c d}} X_{c d}-3 Q_{\alpha \beta} X_{n+1 l} \theta_{l}\right)\left(t \frac{\partial Q_{\gamma \eta}}{\partial B_{e f}} X_{e f}-3 Q_{\gamma \eta} X_{n+1 s} \theta_{s}\right)$

$$
\begin{aligned}
& -2 \frac{F^{\alpha \beta, p_{l}}}{t^{6}} \theta_{l}\left(t \frac{\partial Q_{\alpha \beta}}{\partial B_{c d}} X_{c d}-3 Q_{\alpha \beta} X_{n+1 r} \theta_{r}\right) X_{n+1 s} \theta_{s} \\
& +\frac{F^{\alpha \beta}}{t^{3}} \frac{\partial^{2} Q_{\alpha \beta}}{\partial B_{c d} \partial B_{e f}} X_{c d} X_{e f}-6 \frac{F^{\alpha \beta} Q_{\alpha \beta}}{t^{5}} X_{n+1 l} X_{n+1 s} \theta_{l} \theta_{s}
\end{aligned}
$$$$
-6 \frac{F^{\alpha \beta}}{t^{5}}\left(t \frac{\partial Q_{\alpha \beta}}{\partial B_{c d}} X_{c d}-3 Q_{\alpha \beta} X_{n+1 r} \theta_{r}\right) X_{n+1 s} \theta_{s}
$$

$$
\begin{aligned}
& +2 \frac{F^{p_{s}} \theta_{s}}{t^{3}} X_{n+1 l} X_{n+1 r} \theta_{l} \theta_{r}+\frac{F^{p_{s}, p_{m}} \theta_{s} \theta_{m}}{t^{4}} X_{n+1 l} X_{n+1 r} \theta_{l} \theta_{r}+F^{x_{k}, x_{l}} Z_{k} Z_{l} \\
& +2 \frac{F^{\alpha \beta, x_{k}}}{t^{4}}\left(t \frac{\partial Q_{\alpha \beta}}{\partial B_{c d}} X_{c d}-3 Q_{\alpha \beta} X_{n+1 r} \theta_{r}\right) Z_{k}-2 \frac{F^{u_{l}, x_{k}} \theta_{l}}{t^{2}} X_{n+1 s} \theta_{s} Z_{k} .
\end{aligned}
$$

This expression suggests us to set

$$
\tilde{X}_{\alpha \beta}=t^{-4}\left(\sum_{c, d} t \frac{\partial Q_{\alpha \beta}}{\partial B_{c d}} X_{c d}-3 Q_{\alpha \beta} \sum_{l} X_{n+1 l} \theta_{l}\right), \quad \tilde{Y}=-t^{-2} \sum_{s} X_{n+1 s} \theta_{s}
$$

For

$$
V=\left(\left(X_{c d}\right),\left(Z_{k}\right)\right), H(V, V)=V \nabla_{(\mathbf{B}, x)}^{2} F V^{T}
$$


$H(V, V)$ can be written as

$$
\begin{aligned}
H(V, V)= & F^{\alpha \beta, \gamma \eta} \widetilde{X}_{\alpha \beta} \widetilde{X}_{\gamma \eta}+2 F^{\alpha \beta, p_{l}} \theta_{l} \widetilde{X}_{\alpha \beta} \widetilde{Y}+2 F^{\alpha \beta, x_{k}} \widetilde{X}_{\alpha \beta} Z_{k} \\
& +F^{p_{l}, p_{s}} \theta_{l} \theta_{s} \widetilde{Y}^{2}+2 F^{p_{l}, x_{k}} \theta_{l} \widetilde{Y} Z_{k}+F^{x_{k}, x_{l}} Z_{k} Z_{l}+2 t F^{p_{l}} \theta_{l} \widetilde{Y}^{2} \\
& +6 t F^{\alpha \beta} \widetilde{X}_{\alpha \beta} \widetilde{Y}-6 t^{-1} F^{\alpha \beta} Q_{\alpha \beta} \widetilde{Y}^{2}+\frac{I}{t^{3}}
\end{aligned}
$$

where Einstein summation convention is used and $I$ is defined in (2.5). At this point, we have proved

Lemma 2.3. Condition (1.8) is equivalent to $H(V, V) \leqslant 0, \forall V=\left(\left(X_{c d}\right),\left(Z_{k}\right)\right) \in \mathcal{T} \Gamma_{F}$, where $F^{\alpha \beta, r s}, F^{\alpha \beta, p_{l}}$, etc. in (2.10) are evaluated at $\left(t^{-3} Q, t^{-1} \theta, u, x\right)$.

We may now compare condition (1.8) and (1.6), these are the two structural conditions introduced in [3] (Condition (3.10) and Condition (1.2) there). As already discussed by Bianchini-Longinetti-Salani in [3], a variation of these two conditions can be compared (Theorem 3.12 in [3]). In fact, the following is true.

Corollary 2.4. The condition (1.6) that $\Xi_{F}$ for each $\theta$, $u$ is locally convex implies condition (1.8).

Proof. Lemma 4.1 in [2] states that condition (1.6) implies $H(V, V) \leqslant t^{-3} I$ where $H(V, V)$ is defined in (2.10) and $I$ is defined in (2.5) respectively. The corollary follows directly from lemma 2.2 and lemma 2.3 .

The quantity $I$ defined in (2.5) is a crucial term. We wish to compute this term explicitly, so it can be used in the proof of main theorems in the last section. For our purpose, we set $\theta=(0, \cdots, 0,1)$. In this case, $\mathbf{A}$ and $\mathbf{B}$ can be written as (see [3])

$$
\begin{aligned}
\mathbf{A} & =\left(\begin{array}{ccccc} 
& & & \times & 0 \\
& a_{i j} & & \vdots & \vdots \\
& & & \times & 0 \\
\times & \cdots & \times & \times & \mu \\
0 & \cdots & 0 & \mu & 0
\end{array}\right), \\
\mathbf{B} & =\left(\begin{array}{ccccc} 
& & & 0 & \times \\
& a^{i j} & & \vdots & \vdots \\
& & & 0 & \times \\
0 & \cdots & 0 & 0 & t \\
\times & \cdots & \times & t & \chi
\end{array}\right)
\end{aligned}
$$

where the $(n-1) \times(n-1)$ matrix $\left(a_{i j}\right)$ is negative definite and can be assumed diagonal, $\left(a^{i j}\right)$ is the inverse matrix of $\left(a_{i j}\right), t=B_{n+1, n}=\frac{1}{\mu}>0$. The values at the positions denoted by $\times$ which are not important in the calculations.

Note that $B_{l n}=B_{n l} \equiv 0, \forall l \leqslant n$. We may as well set

$$
X_{l n}=X_{n l}=0, \forall l \leqslant n .
$$


Denote

$$
B^{\alpha \beta}=\left(B^{-1}\right)_{\alpha \beta}=A_{\alpha \beta}, \quad T:=\{1, \ldots, n-1\}
$$

We compute

$$
\begin{aligned}
& F^{\alpha \beta} \frac{\partial^{2} Q_{\alpha \beta}}{\partial B_{c d} \partial B_{e f}} X_{c d} X_{e f} \\
= & 2 t^{2} F^{\alpha \beta} B^{\alpha e} B^{f c} B^{d \beta} X_{c d} X_{e f}-4 t F^{\alpha \beta} B^{\alpha c} B^{d \beta} X_{c d} X_{n+1 n}+2 F^{\alpha \beta} B^{\alpha \beta} X_{n+1 n}^{2},
\end{aligned}
$$

by breaking summation into the following three parts.

Case 1. $\alpha, \beta \in T$. We can see if $c=n$, then $d$ must be $n+1$, as $B^{n+1 \beta}=0$,

$$
\begin{aligned}
& 2 \sum_{i \in T} t^{2} B^{\alpha \alpha} B^{i i} B^{\beta \beta} X_{\alpha i} X_{\beta i}-4 t B^{\alpha \alpha} B^{\beta \beta} X_{\alpha \beta} X_{n+1 n}+2 F^{\alpha \alpha} B^{\alpha \alpha} X_{n+1 n}^{2} \\
= & \sum_{i \in T} \frac{2}{B^{i i}}\left(t B^{i i} B^{\alpha \alpha} X_{i \alpha}-B^{i \alpha} X_{n+1 n}\right)\left(t B^{i i} B^{\beta \beta} X_{i \beta}-B^{i \beta} X_{n+1 n}\right) .
\end{aligned}
$$

Case 2. $\alpha=n, \beta \in T$ or $\beta=n, \alpha \in T$. As $B^{n n+1}=\frac{1}{t}$,

$$
\begin{aligned}
& 2 \sum_{i \in T} t^{2} B^{\alpha \alpha} B^{i i} B^{\beta \beta} X_{\alpha i} X_{\beta i}-4 t B^{\alpha \alpha} B^{\beta \beta} X_{\alpha \beta} X_{n+1 n}+2 F^{\alpha \alpha} B^{\alpha \alpha} X_{n+1 n}^{2} \\
& =2 t^{2} B^{n e} B^{f c}\left(B^{\beta \beta} X_{c \beta}+B^{n \beta} X_{c n}\right) X_{e f} \\
& -4 t B^{n c}\left(B^{\beta \beta} X_{c \beta}+B^{n \beta} X_{c n}\right) X_{n+1 n}+2 B^{n \beta} X_{n+1 n}^{2} \\
& =2 t^{2} B^{n e}\left(\sum_{c \neq n} B^{f c} B^{\beta \beta} X_{c \beta}+B^{f n+1} B^{n \beta} X_{n+1 n}\right) X_{e f} \\
& -4 t\left(\sum_{c \neq n} B^{n c} B^{\beta \beta} X_{c \beta}+B^{n n+1} B^{n \beta} X_{n+1 n}\right) X_{n+1 n}+2 B^{n \beta} X_{n+1 n}^{2} \\
& =2 t^{2} B^{n e} \sum_{c \neq n} B^{f c} B^{\beta \beta} X_{c \beta} X_{e f}-4 t \sum_{c \neq n} B^{n c} B^{\beta \beta} X_{c \beta} X_{n+1 n} \\
& =2 t^{2} \sum_{c \neq n}\left(\sum_{f \neq n} B^{n e} B^{f c} B^{\beta \beta} X_{c \beta} X_{e f}+B^{n n+1} B^{n c} B^{\beta \beta} X_{c \beta} X_{n+1 n}\right) \\
& -4 t \sum_{c \neq n} B^{n c} B^{\beta \beta} X_{c \beta} X_{n+1 n} \\
& =2 t^{2} \sum_{e \neq n} \sum_{i \in T} B^{n e} B^{i i} B^{\beta \beta} X_{i \beta} X_{i e}-2 t \sum_{c \neq n} B^{n c} B^{\beta \beta} X_{c \beta} X_{n+1 n} \\
& =2 \sum_{i \in T} \frac{1}{B^{i i}}\left(t B^{i i} B^{\beta \beta} X_{i \beta}-B^{i \beta} X_{n+1 n}\right)\left(t \sum_{c \neq n} B^{i i} B^{n c} X_{c i}\right) \text {. }
\end{aligned}
$$


Case 3. $\alpha=\beta=n$.

$$
\begin{aligned}
& \frac{\partial^{2} Q_{n n}}{\partial B_{c d} \partial B_{e f}} X_{c d} X_{e f} \\
= & 4 t^{2} \sum_{c, d \neq n}\left(B^{n n} B^{n+1 c} B^{d n} X_{c d} X_{n n+1}+B^{n n+1} B^{n c} B^{d n} X_{c d} X_{n+1 n}\right) \\
& +2 t^{2} \sum_{c, d, e, f \neq n} B^{n e} B^{f c} B^{d n} X_{c d} X_{e f}+2 t^{2} B^{n n}\left(B^{n n+1} B^{n+1 n} X_{n n+1} X_{n+1 n}\right. \\
& \left.+B^{n+1 n+1} B^{n n} X_{n+1 n} X_{n n+1}+B^{n+1 n} B^{n+1 n} X_{n n+1}^{2}+B^{n n+1} B^{n n+1} X_{n+1 n}^{2}\right) \\
& -4 t \sum_{c, d \neq n} B^{n c} B^{d n} X_{c d} X_{n+1 n}-8 t B^{n n} B^{n+1 n} X_{n+1 n}^{2}+2 B^{n n} X_{n+1 n}^{2} .
\end{aligned}
$$

It follows from the facts that $B^{n n+1}=\frac{1}{t}$ and $B^{n+1 \beta}=0, \quad \forall \beta \neq n$,

$$
\begin{aligned}
F^{n n} \frac{\partial^{2} Q_{n n}}{\partial B_{c d} \partial B_{e f}} X_{c d} X_{e f} & =2 t^{2} \sum_{c, d, e, f \neq n} F^{n n} B^{n e} B^{f c} B^{d n} X_{c d} X_{e f} \\
& =2 t^{2} \sum_{i \in T} \sum_{d, e \neq n} F^{n n} B^{n e} B^{i i} B^{d n} X_{i d} X_{e i} \\
& =2 \sum_{i \in T} \frac{F^{n n}}{B^{i i}}\left(t \sum_{e \neq n} B^{n e} B^{i i} X_{e i}\right)^{2} .
\end{aligned}
$$

Set

$$
Y_{i \alpha}:=t B^{i i} B^{\alpha \alpha} X_{i \alpha}-B^{i \alpha} X_{n+1 n}, \forall \alpha \in T ; \quad Y_{i n}:=t B^{i i} \sum_{c \neq n} B^{n c} X_{c i} .
$$

Combining (2.14), (2.15) and (2.16), for $\theta=(0, \cdots, 0,1), I$ in (2.5) can be written as

$$
I=2 \sum_{i \in T} \frac{F^{\alpha \beta}}{B^{i i}} Y_{i \alpha} Y_{i \beta},
$$

where $Y_{i \alpha}, \forall \alpha \in\{1, \ldots, n\}$ is defined in (2.17).

We wish to express $I$ in terms of $\widetilde{X}_{\alpha \beta}$ and A. Recall $Q_{\alpha \beta}=t^{2} B^{\alpha \beta}$,

$$
\begin{gathered}
\frac{\partial Q_{\alpha \beta}}{\partial B_{c d}}=\frac{\partial\left(t^{2} B^{\alpha \beta}\right)}{\partial B_{c d}}=-t^{2} B^{\alpha c} B^{d \beta}+2 t B^{\alpha \beta} \delta_{n+1 c} \delta_{n d} \\
\sum_{c, d} t \frac{\partial Q_{\alpha \beta}}{\partial B_{c d}} X_{c d}-3 Q_{\alpha \beta} \sum_{l} X_{n+1 l} \theta_{l}=-t^{3} \sum_{c, d} B^{\alpha c} B^{d \beta} X_{c d}-t^{2} B^{\alpha \beta} X_{n+1 n}
\end{gathered}
$$

By (2.9)

$$
t^{2} \widetilde{X}_{\alpha \beta}=-t B^{\alpha c} B^{d \beta} X_{c d}-B^{\alpha \beta} X_{n+1, n}, \quad 1 \leqslant \alpha, \beta \leqslant n .
$$


Extending the definition of $\widetilde{X}$ as $(n+1) \times(n+1)$ symmetric matrix by setting

$$
t^{2} \widetilde{X}_{e f}=-t B^{e c} B^{d f} X_{c d}-B^{e f} X_{n+1, n}, \quad 1 \leqslant e, f \leqslant n+1 .
$$

Since $X_{n \alpha}=0, \forall \alpha \leqslant n$, and $B^{n+1, c}=A_{n+1, c}=0, \forall c \neq n$,

$$
\widetilde{X}_{n+1, c}=0, \forall c \neq n ; \quad \widetilde{X}_{n+1, n}=-\frac{2}{t^{3}} X_{n+1, n}=\frac{2}{t} \widetilde{Y} .
$$

In this setting, $X_{c d}$ can be recovered using the formula below,

$$
X_{c d}=-t A^{c e} A^{f d} \widetilde{X}_{e f}+\frac{t^{2} A^{c d}}{2} \widetilde{X}_{n+1, n}, \quad 1 \leqslant c, d \leqslant n+1 .
$$

From the relationship $\mathbf{B}=\mathbf{A}^{-1}$, and the fact that $(n-1) \times(n-1)$-matrix $\left(A_{\alpha \beta}\right)$ is diagonal, we have $B_{n+1, i}=-\frac{t A_{n, i}}{A_{i i}}$. Hence from (2.17),

$$
Y_{i \alpha}=t^{3} A_{i \alpha} \widetilde{X}_{n+1, n}-t^{2} \widetilde{X}_{i \alpha} .
$$

It follows from (2.18) and (2.25),

Lemma 2.5. For $\theta=(0, \cdots, 0,1)$, if $\left(A_{\alpha \beta}\right)$ is diagonal, then $I$ in (2.5) can be written as

$$
I=2 \sum_{i \in T} \frac{F^{\alpha \beta}}{A_{i i}} Y_{i \alpha} Y_{i \beta},
$$

where $Y_{i \alpha}$ is defined in (2.25).

From (2.6), (2.7) and (2.9),

$$
<V, \nabla_{(B, x)} F>=F^{\alpha \beta} \widetilde{X}_{\alpha \beta}+F^{u_{n}} \tilde{Y}+F^{x_{k}} Z_{k}
$$

$V=\left(\left(X_{c d}\right),\left(Z_{k}\right)\right) \in \mathcal{T} \Gamma_{F}$ if and only if

$$
F^{\alpha \beta} \widetilde{X}_{\alpha \beta}+F^{u_{n}} \tilde{Y}+F^{x_{k}} Z_{k}=0
$$

where $\widetilde{X}_{\alpha \beta}, \tilde{Y}$ as in (2.21) and (2.23) respectively, and $F^{\alpha \beta}, F^{u_{n}}$ and $F^{x_{k}}$ are evaluated at $\left(t^{-1} \widetilde{\mathbf{A}}, t^{-1} \theta, u, x\right)$ with $\theta=(0, \cdots, 0,1)$.

Set $\widetilde{V}=\left(\left(\widetilde{X}_{c d}\right),\left(Z_{k}\right)\right)$ where $\left(\widetilde{X}_{c d}\right)$ defined by (2.22) with (2.23) , rewrite (2.10) as

$$
H(\widetilde{V}, \widetilde{V})=\frac{I}{t^{3}}+S,
$$

where $I$ is defined as in (2.26),

$$
\begin{aligned}
S= & F^{\alpha \beta, \gamma \eta} \widetilde{X}_{\alpha \beta} \widetilde{X}_{\gamma \eta}+2 F^{\alpha \beta, p_{n}} \widetilde{X}_{\alpha \beta} \widetilde{Y}+2 F^{\alpha \beta, x_{k}} \widetilde{X}_{\alpha \beta} Z_{k}+F^{p_{n}, p_{n}} \widetilde{Y}^{2} \\
& +2 F^{p_{n}, x_{k}} \widetilde{Y} Z_{k}+F^{x_{k}, x_{l}} Z_{k} Z_{l}+2 t F^{p_{n}} \widetilde{Y}^{2}+6 t F^{\alpha \beta} \widetilde{X}_{\alpha \beta} \widetilde{Y}-6 t F^{\alpha \beta} A_{\alpha \beta} \widetilde{Y}^{2},
\end{aligned}
$$

and $F^{\alpha \beta}, F^{u_{n}}, F^{x_{k}}$ etc. are evaluated at $\left(t^{-1} \widetilde{\mathbf{A}}, t^{-1} \theta, u, x\right), \widetilde{\mathbf{A}} \in \mathscr{S}_{n}^{-}(\theta) \cap \Upsilon$.

By (2.24) and Lemma 2.3. 
Lemma 2.6. Condition (1.8) is equivalent to

$$
H(\widetilde{V}, \widetilde{V}) \leqslant 0, \forall \tilde{V}=\left(\left(\widetilde{X}_{c d}\right),\left(Z_{k}\right)\right) \text { satisfying (2.27). }
$$

By approximation, if Condition (1.8) is satisfied, then

$$
H(\widetilde{V}, \widetilde{V}) \leqslant 0,
$$

for every $\tilde{V}=\left(\left(\widetilde{X}_{c d}\right),\left(Z_{k}\right)\right)$ satisfying 2.27$)$ at each $\widetilde{\mathbf{A}} \in \overline{\mathscr{S}_{n}^{-}(\theta)} \cap \Upsilon$ diagonal, where $\overline{\mathscr{S}_{n}^{-}(\theta)}$ is the closure of $\mathscr{S}_{n}^{-}(\theta)$, with $Y_{k \alpha}=0$ when $a_{k k}=0$ for some $k \leqslant n-1$.

With the explicit expression of $H$ in Lemma 2.6, we may verify condition (1.8) for mean curvature operator and general quasilinear operator $F$ satisfying structural conditions in [14]. Condition (1.6) is not satisfied by mean curvature operator as indicated in [3]. It was verified there that for $n=2$, the Mean Curvature operator:

$$
F\left(D^{2} u, D u\right)=\operatorname{Div}\left(\frac{D u}{\sqrt{1+|D u|^{2}}}\right)=\frac{\Delta u}{\sqrt{1+|D u|^{2}}}-\sum_{\alpha, \beta=1}^{n} \frac{u_{\alpha} u_{\beta} u_{\alpha \beta}}{{\sqrt{1+|D u|^{2}}}^{3}}=f(u) \geqslant 0,
$$

satisfies condition (1.8), but not (1.6). Here we verify this fact for general $n$. Since condition (1.8) and (1.6) are invariant under orthogonal transformation, we may as well set $D u=\left(0, \ldots, 0, u_{n}\right),\left(u_{i j}\right)$ is diagonal for each $i, j \in T=\{1, \ldots, n-1\}$. We also note $\left(u_{i j}\right)$ is negative definite. According to (1.4), $t^{-1} \theta=D u$, where $\theta=(0, \ldots, 0,1)$ and $t^{-1}=u_{n}$, $\mathbf{J} B^{-1} \mathbf{J}^{T}=u_{n}^{-1}\left(D^{2} u\right)$. Since the mean Curvature operator $F$ in (2.30) is homogenous of one degree, $S$ in (2.29) can be calculated as

$$
S=2 F^{\alpha \beta, u_{n}} \widetilde{X}_{\alpha \beta} \tilde{Y}+F^{u_{n}, u_{n}} \widetilde{Y}^{2}+2 \frac{F^{u_{n}}}{u_{n}} \widetilde{Y}^{2}+6 \frac{F^{\alpha \beta}}{u_{n}} \widetilde{X}_{\alpha \beta} \widetilde{Y}-6 u_{n}^{-2} F \widetilde{Y}^{2}
$$

From (2.27), $\widetilde{V}=\left(\left(\widetilde{X}_{c d}\right)\right)$ satisfies

$$
0=\left\langle\widetilde{V}, \nabla_{B} F\right\rangle=F^{\alpha \beta} \widetilde{X}_{\alpha \beta}+F^{u_{n}} \widetilde{Y} .
$$

A straightforward calculation yields that

$$
S=\sum_{i \in T}\left(\frac{4 u_{n}}{W^{3}} \widetilde{X}_{i i} \tilde{Y}-\frac{6}{W^{3}} u_{i i} \widetilde{Y}^{2}\right)-\frac{3+6 u_{n}^{-2}}{W^{4}} F \widetilde{Y}^{2}
$$

where $W=\sqrt{1+|D u|^{2}}$. It is easy to check that $S \leqslant 0$ is violated for some $\widetilde{X}_{i i}, \widetilde{Y}$ satisfying (2.32). On the other hand, Lemma 4.1 in [2] implies that $S \leqslant 0$ if condition [1.6 is satisfied. Therefore, $F$ does not satisfy condition (1.6).

However, from (2.26)

$$
I=2 u_{n} \sum_{i \in T} \frac{F^{\alpha \beta}}{u_{i i}} Y_{i \alpha} Y_{i \beta}
$$

For the mean curvature equation, it can be computed that

$$
Y_{i \alpha}=-t^{2} \widetilde{X}_{i \alpha}+2 t^{2} u_{i \alpha} \tilde{Y} u_{n}^{-1}, \quad \forall i, \alpha \in G .
$$


By (2.33), (2.34) and (2.35), and the facts that $u_{i i}<0, F \geqslant 0$,

$$
\begin{aligned}
H & =S+I u_{n}^{3} \\
& \leqslant S+2 u_{n}^{4} \sum_{i \in T} \frac{F^{i i}}{u_{i i}} Y_{i i}^{2} \\
& =2 \sum_{i \in T}\left(\frac{1}{W u_{i i}} \widetilde{X}_{i i}^{2}-2 \frac{u_{n}+2 u_{n}^{-1}}{W^{3}} \widetilde{X}_{i i} \tilde{Y}+\frac{4 u_{n}^{-2}+1}{W^{3}} u_{i i} \widetilde{Y}^{2}\right)-\frac{3+6 u_{n}^{-2}}{W^{4}} F \widetilde{Y}^{2} \\
& =\sum_{i \in T} \frac{2}{W u_{i i}}\left[\left(\widetilde{X}_{i i}^{2}-\frac{u_{n}+2 u_{n}^{-1}}{W^{2}} u_{i i} \widetilde{Y}\right)^{2}+\frac{1}{W^{4}} u_{i i}^{2} \widetilde{Y}^{2}\right]-\frac{3+6 u_{n}^{-2}}{W^{4}} F \widetilde{Y}^{2} \\
& \leqslant 0
\end{aligned}
$$

That is the mean curvature operator $F$ satisfies condition (1.8) by Lemma 2.6. This example indicates that the term $I$ is the key. The verification of condition (1.8) for the quasilinear operators considered in [11, 14] can be done in a similar way, we leave for the interested reader to check them.

\section{THE TEST FUNCTION}

The proof of our main results relies on the establishment of a maximum principle for certain appropriate curvature test function. This section is devoted to discuss some regularity and concavity properties of the proposed test function.

We will assume $u \in C^{3,1}(\Omega),|\nabla u|>0$ and $\{x \in \Omega \mid u(x) \geqslant c\} \cup \Omega_{1}$ is locally convex in the rest of this paper.

We recall some of formulas related to the Weingarten curvature tensor of level surfaces. Suppose $u$ is a function defined in an open set in $\mathbb{R}^{n}$, assume that $u_{n}(x) \neq 0$. The upward inner normal direction of the level sets of $u$ is

$$
\vec{n}=\frac{\left|u_{n}\right|}{|D u| u_{n}}\left(u_{1}, u_{2}, \ldots, u_{n-1}, u_{n}\right) .
$$

It's calculated in [2] that the second fundamental form $I I$ of the level surface of function $u$ with respect to the upward normal direction (3.1) is

$$
h_{i j}=-\frac{\left|u_{n}\right|\left(u_{n}^{2} u_{i j}+u_{n n} u_{i} u_{j}-u_{n} u_{j} u_{i n}-u_{n} u_{i} u_{j n}\right)}{|D u| u_{n}^{3}}, \quad i, j \leqslant n-1 .
$$

Note that as $\{x \in \Omega \mid u(x) \geqslant c\} \cup \Omega_{1}$ is locally convex, the second fundamental form of $\Sigma^{c}$ is nonnegative definite with respect to the upward normal direction (3.1). For $D u$ is as the same direction as $\vec{n}$, thus we have $u_{n}>0$ locally. (3.2) implies that the matrix $\left(u_{i j}(x)\right)$ is nonpositive definite.

Denote $a(x)=\left(a_{i j}(x)\right)$ the symmetric Weingarten tensor of $\Sigma^{u(x)}=\{y \in \Omega \mid u(y)=$ $u(x)\}$. Our assumption implies that $a$ is nonnegative definite. Since $u_{n}(x) \neq 0$, following [4, the Weingarten tensor can be computed as (see [2]),

$$
a_{i j}=h_{i j}-\frac{\sum_{l=1}^{n-1} u_{i} u_{l} h_{j l}}{W(1+W) u_{n}^{2}}-\frac{\sum_{l=1}^{n-1} u_{j} u_{l} h_{i l}}{W(1+W) u_{n}^{2}}+\frac{\sum_{l, k=1}^{n-1} u_{i} u_{j} u_{k} u_{l} h_{k l}}{W^{2}(1+W)^{2} u_{n}^{4}}, \quad i, j \leqslant n-1,
$$


where $W=\left(1+\left|\nabla_{x^{\prime}} v\right|^{2}\right)^{\frac{1}{2}}$ and $x^{\prime}=\left(x_{1}, \cdots, x_{n-1}\right)$.

Set

$$
\widetilde{a}=a-\eta_{0} g(u) I, \quad \eta_{0} \geqslant 0, \quad g(u)=e^{A u}
$$

where $\eta_{0} \geqslant 0$ and $A \geqslant 0$ are constants to be determined later such that $\widetilde{a} \geqslant 0$.

Suppose the minimal $\operatorname{rank} l$ of $\widetilde{a}$ is attained at some interior point $x_{0}$. Let $\mathcal{O}$ be a small open neighborhood of $x_{0}$ such that for each $x \in \mathcal{O}$, there are $l$ "good" eigenvalues of $\left(\widetilde{a}_{i j}\right)$ which are bounded below by a positive constant, and the other $n-1-l$ "bad" eigenvalues of $\left(\widetilde{a}_{i j}\right)$ are very small. Denote $G$ be the index set of these "good" eigenvalues and $B$ be the index set of "bad" eigenvalues. For each $x \in \mathcal{O}$ fixed, we may express $\left(a_{i j}\right)$ in a form of (3.3), by choosing $e_{1}, \cdots, e_{n-1}, e_{n}$ such that

$$
|D u|(x)=u_{n}(x)>0,\left(u_{i j}(x)\right), i, j=1, . ., n-1 \text { is diagonal. }
$$

From (3.3) and (3.4), the matrix $\left(\widetilde{a}_{i j}\right), i, j=1, . ., n-1$ is also diagonal at $x$, and without loss of generality we may assume $\widetilde{a}_{11} \leqslant \widetilde{a}_{22} \leqslant \ldots \leqslant \widetilde{a}_{n-1, n-1}$. There is a positive constant $C>0$ depending only on $\|u\|_{C^{4}}$ and $\mathcal{O}$, such that $\widetilde{a}_{n-1, n-1} \geqslant \widetilde{a}_{n-2, n-2} \geqslant \ldots \geqslant \widetilde{a}_{n-l, n-l}>C$ for all $x \in \mathcal{O}$. For convenience we denote $G=\{n-l, n-l+1, \ldots, n-1\}$ and $B=\{1,2, \ldots, n-l-1\}$ be the "good" and "bad" sets of indices respectively. If there is no confusion, we also denote

$$
B=\left\{\widetilde{a}_{11}, \ldots, \widetilde{a}_{n-l-1, n-l-1}\right\} \text { and } G=\left\{\widetilde{a}_{n-l, n-l}, \ldots, \widetilde{a}_{n-1, n-1}\right\} .
$$

Note that for any $\delta>0$, we may choose $\mathcal{O}$ small enough such that $\widetilde{a}_{j j}<\delta$ for all $j \in B$ and $x \in \mathcal{O}$.

The following two functions are of fundamental importance in our treatment.

$$
p(\widetilde{a})=\sigma_{l+1}\left(\widetilde{a}_{i j}\right), \quad q(\widetilde{a})= \begin{cases}\frac{\sigma_{l+2}\left(\widetilde{a}_{i j}\right)}{\sigma_{l+1}\left(\widetilde{a}_{i j}\right)}, & \text { if } \sigma_{l+1}\left(\widetilde{a}_{i j}\right)>0 \\ 0, & \text { otherwise. }\end{cases}
$$

We consider function

$$
\phi(\widetilde{a})=p(\widetilde{a})+q(\widetilde{a})
$$

where $p$ and $q$ as in (3.7). The function $\phi$ was first introduced in [1] for the Hessian of solution $u$, and for Weingarten tensor $a$ in [2]. Here we adopt it as a function in $\widetilde{a}$.

We will use notion $h=O(f)$ if $|h(x)| \leqslant C f(x)$ for $x \in \mathcal{O}$ with positive constant $C$ under control. Again, as in [1], to get around $p=0$, for $\varepsilon>0$ sufficiently small, we instead consider

$$
\phi_{\varepsilon}(\widetilde{a})=\phi\left(\widetilde{a}_{\varepsilon}\right),
$$

where $\widetilde{a}_{\varepsilon}=\widetilde{a}+\varepsilon I$. We will also denote $G_{\varepsilon}=\left\{\widetilde{a}_{i i}+\varepsilon, i \in G\right\}, B_{\varepsilon}=\left\{\widetilde{a}_{i i}+\varepsilon, i \in B\right\}$.

We will write $p$ for $p_{\varepsilon}, \phi$ for $\phi_{\varepsilon}, q$ for $q_{\varepsilon}, \widetilde{a}$ for $\widetilde{a}_{\varepsilon}, G$ for $G_{\varepsilon}, B$ for $B_{\varepsilon}$ with the understanding that all the estimates will be independent of $\varepsilon$. In this setting, if we pick $\mathcal{O}$ small enough, there is $C>0$ independent of $\varepsilon$ such that

$$
\phi(\widetilde{a}(z)) \geqslant C \varepsilon, \quad \sigma_{1}(B(z)) \geqslant C \varepsilon, \text { for all } \mathrm{z} \in \mathcal{O} .
$$


In what follows, $i, j, \cdots$ will be denoted as indices run from 1 to $n-1$ and the Greek indices $\alpha, \beta, \cdots$ will be denoted as indices run from 1 to $n$. Denote

$$
p_{\alpha}=\frac{\partial p}{\partial x_{\alpha}}, \quad p_{\alpha \beta}=\frac{\partial^{2} p}{\partial x_{\alpha} \partial x_{\beta}}, \quad F^{\alpha \beta}=\frac{\partial F}{\partial u_{\alpha \beta}}, \quad 1 \leqslant \alpha, \beta \leqslant n
$$

We also denote $g=e^{A u}$,

$$
\mathcal{H}_{\phi}=\sum_{i, j \in B}\left|\nabla \widetilde{a}_{i j}\right|+\phi
$$

and $\forall j \in B$,

$$
I_{j}=\sum_{i \in G}\left[-2 u_{n}^{3} \sum_{\alpha, \beta \notin B} \frac{F^{\alpha \beta} a_{i j, \alpha} a_{i j, \beta}}{a_{i i}}+4 u_{n}^{2} u_{n j} \sum_{\alpha \notin B} F^{\alpha i} a_{i j, \alpha}+2 u_{n j}^{2} F^{i i} u_{i i}\right]
$$

and

$$
\begin{aligned}
J_{1 j}= & -12 \sum_{\alpha=1}^{n} F^{j \alpha} u_{n \alpha} u_{j n} u_{j j}+4 \sum_{\alpha=1}^{n} F^{j \alpha} u_{j n \alpha} u_{n} u_{j j}-2 u_{n n} F^{j j} u_{j j}^{2} \\
& -\eta_{0} \sum_{\alpha, \beta=1}^{n} F^{\alpha \beta} g_{\alpha \beta} u_{n}^{3}+4 \eta_{0} \sum_{\alpha=1}^{n} F^{j \alpha} g_{\alpha} u_{j n} u_{n}^{2}-2 \eta_{0} \sum_{\alpha, \beta=1}^{n} F^{\alpha \beta} u_{n \alpha} g_{\beta} u_{n} \\
& -\eta_{0} g \sum_{\alpha, \beta=1}^{n} F^{\alpha \beta}\left(2 u_{j \alpha} u_{j \beta} u_{n}+u_{n \alpha \beta} u_{n}^{2}+\sum_{i=1}^{n-1} u_{i \alpha} u_{i \beta} u_{n}\right)-2 \eta_{0} g\left(\sum_{i \in B} F^{i i}\right) u_{n j}^{2} u_{n} \\
& -2 \eta_{0} g \sum_{\alpha, \beta=1}^{n} \sum_{i \in G} \frac{F^{\alpha \beta} a_{i j, \alpha} a_{i j, \beta}}{a_{i i} \widetilde{a}_{i i}} u_{n}^{3},
\end{aligned}
$$

and

$$
\begin{aligned}
J_{2 j}= & 2 F^{\alpha \beta, u_{j}} u_{j \alpha \beta} u_{j j}+2 F^{u_{n} u_{j}} u_{j n} u_{j j}+2 F^{u_{j}, x_{j}} u_{j j}+F^{u} u_{j j} \\
& +F^{u_{l}} u_{l n} u_{n}^{-1} u_{j j}+2 F^{u_{j}} u_{j n} u_{n}^{-1} u_{j j}+F^{u_{j} u_{j}} u_{j j}^{2}-\eta_{0} F^{u_{l}} g_{l} u_{n} .
\end{aligned}
$$

Lemma 3.1. Suppose $u \in C^{3,1}$ is a solution of equation (1.1) with $|\nabla u|>0$, then $\phi \in$ $C^{1,1}(\mathcal{O})$. For any fixed $x \in \mathcal{O}$, with the coordinate chosen as in (3.5) and (3.6),

$$
\phi_{\alpha}=\left[\sigma_{l}(G)+\frac{\sigma_{1}^{2}(B \mid j)-\sigma_{2}(B \mid j)}{\sigma_{1}^{2}(B)}\right] \widetilde{a}_{j j, \alpha}+O\left(\mathcal{H}_{\phi}\right)
$$


and

$$
\begin{aligned}
& F^{\alpha \beta} \phi_{\alpha \beta} \\
= & \sum_{j \in B} u_{n}^{-3}\left[\sigma_{l}(G)+\frac{\sigma_{1}^{2}(B \mid j)-\sigma_{2}(B \mid j)}{\sigma_{1}^{2}(B)}\right]\left\{\left[\sum_{\alpha, \beta, \gamma, \eta=1}^{n} F^{\alpha \beta, \gamma \eta} u_{\alpha \beta j} u_{\gamma \eta j}\right.\right. \\
& +2 \sum_{\alpha, \beta=1}^{n} F^{\alpha \beta, u_{n}} u_{j \alpha \beta} u_{j n}+2 \sum_{\alpha, \beta=1}^{n} F^{\alpha \beta, x_{j}} u_{j \alpha \beta}+F^{u_{n}, u_{n}} u_{j n}^{2}+2 F^{u_{n}, x_{j}} u_{j n}+F^{x_{j}, x_{j}} \\
& \left.\left.+2 \frac{F^{u_{n}}}{u_{n}} u_{j n}^{2}\right] u_{n}^{2}+6 \sum_{\alpha, \beta=1}^{n} F^{\alpha \beta} u_{j \alpha \beta} u_{j n} u_{n}-6 \sum_{\alpha, \beta=1}^{n} F^{\alpha \beta} u_{\alpha \beta} u_{j n}^{2}+I_{j}+J_{1 j}+J_{2 j}\right\} \\
& -\frac{1}{\sigma_{1}^{3}(B)} \sum_{\alpha, \beta=1}^{n} \sum_{i \in B} F^{\alpha \beta}\left[\sigma_{1}(B) \widetilde{a}_{i i, \alpha}-\widetilde{a}_{i i} \sum_{j \in B} \widetilde{a}_{j j, \alpha}\right]\left[\sigma_{1}(B) \widetilde{a}_{i i, \beta}-\widetilde{a}_{i i} \sum_{j \in B} \widetilde{a}_{j j, \beta}\right] \\
& -\frac{1}{\sigma_{1}(B)} \sum_{\alpha, \beta=1}^{n} \sum_{i \neq j, i, j \in B} F^{\alpha \beta} \widetilde{a}_{i j, \alpha} \widetilde{a}_{i j, \beta}+O\left(\mathcal{H}_{\phi}\right) .
\end{aligned}
$$

$I_{j}, J_{1 j}$ and $J_{2 j}$ in (3.16) are crucial terms. Some fine analysis of these terms are the key in our proof of the main results.

Proof of Lemma 3.1. For any fixed point $x \in \mathcal{O}$, choose a coordinate system as in (3.5) so that $|D u(x)|=u_{n}(x)>0$ and the matrix $\left(\widetilde{a}_{i j}(x)\right)$ is diagonal for $1 \leqslant i, j \leqslant n-1$ and nonnegative. From the definition of $p$,

$$
-\frac{u_{j j}}{u_{n}}-\eta_{0} g=\widetilde{a}_{j j}=O\left(\mathcal{H}_{\phi}\right), \forall j \in B ; \quad p_{\alpha}=\sigma_{l}(G) \sum_{j \in B} \widetilde{a}_{j j, \alpha}+O(\phi) .
$$

By (3.17),

$$
\begin{aligned}
p_{\alpha \beta} & =\sigma_{l}(G)\left[\sum_{j \in B} \widetilde{a}_{j j, \alpha \beta}-2 \sum_{i \in G, j \in B} \frac{\widetilde{a}_{i j, \alpha} \widetilde{a}_{i j, \beta}}{\widetilde{a}_{i i}}\right]+O\left(\mathcal{H}_{\phi}\right) \\
& =\sigma_{l}(G)\left[\sum_{j \in B}\left(a_{j j, \alpha \beta}-\eta_{0} g_{\alpha \beta}\right)-2 \sum_{i \in G, j \in B} \frac{\widetilde{a}_{i j, \alpha} \widetilde{a}_{i j, \beta}}{\widetilde{a}_{i i}}\right]+O\left(\mathcal{H}_{\phi}\right) .
\end{aligned}
$$

Since $u_{k}=0$ at $x$ for $k=1, \cdots, n-1$, from (3.3), and for each $j \in B$,

$$
\begin{aligned}
u_{n}^{3} a_{j j, \alpha \beta}= & -u_{n}^{2} u_{j j \alpha \beta}-2 u_{n}\left(u_{n \beta} u_{j j \alpha}+u_{n \alpha} u_{j j \beta}\right)+2 u_{n}\left(u_{j \alpha} u_{n j \beta}+u_{j \beta} u_{n j \alpha}\right) \\
& +2 u_{n} u_{n j} u_{\alpha \beta j}+2 u_{n j}\left(u_{n \alpha} u_{j \beta}+u_{n \beta} u_{j \alpha}\right)-2 u_{n n} u_{j \alpha} u_{j \beta}-\left(2 u_{n \alpha} u_{n \beta}+\right. \\
& \left.2 u_{n} u_{\alpha \beta n}\right) u_{j j}-2 \eta_{0} g u_{j \alpha} u_{j \beta} u_{n}-3 \eta_{0} u_{n}^{2}\left(u_{n \alpha} g_{\beta}+u_{n \beta} g_{\alpha}\right) \\
& -\eta_{0} g\left(3 u_{n}^{2} u_{n \alpha \beta}+6 u_{n \alpha} u_{n \beta} u_{n}+\sum_{i=1}^{n-1} u_{i \alpha} u_{i \beta} u_{n}\right)+O\left(\mathcal{H}_{\phi}\right) .
\end{aligned}
$$


From the definition of $a_{i j}$,

$$
u_{n} u_{i j \alpha}=-u_{n}^{2} a_{i j, \alpha}+u_{n j} u_{i \alpha}+u_{n i} u_{j \alpha}+u_{n \alpha} u_{i j}, \quad \forall i, j \leqslant n-1,
$$

and

$$
\tilde{a}_{i j, \alpha}=a_{i j \alpha}-\eta_{0} g_{\alpha} \delta_{i j}, \quad \forall i, j \in B
$$

$$
\begin{aligned}
& \sum_{\alpha, \beta=1}^{n} F^{\alpha \beta} a_{j j, \alpha \beta} \\
= & \sum_{\alpha, \beta=1}^{n} \frac{F^{\alpha \beta}}{u_{n}^{3}}\left[-u_{n}^{2} u_{\alpha \beta j j}-4 u_{n \alpha} u_{n j} u_{j \beta}+4 u_{n} u_{j \alpha} u_{n j \beta}+2 u_{n} u_{n j} u_{\alpha \beta j}-2 u_{n n} u_{j \alpha} u_{j \beta}\right. \\
& \left.-\eta_{0} g\left(2 u_{j \alpha} u_{j \beta} u_{n}+u_{n \alpha \beta} u_{n}^{2}+\sum_{i=1}^{n-1} u_{i \alpha} u_{i \beta} u_{n}\right)-2 \eta_{0} u_{n \alpha} g_{\beta} u_{n}^{2}\right]+O\left(\mathcal{H}_{\phi}\right) .
\end{aligned}
$$

Break summation as $\sum_{\alpha=1}^{n} F^{\alpha n} u_{n \alpha}=\left(\sum_{\alpha, \beta=1}^{n}-\sum_{\beta=1}^{n-1} \sum_{\alpha=1}^{n}\right) F^{\alpha \beta} u_{\alpha \beta}, \forall j \in B$,

$$
\begin{aligned}
& \sum_{\alpha, \beta=1}^{n} F^{\alpha \beta} u_{n \alpha} u_{j \beta}=u_{n j}\left(\sum_{\alpha, \beta=1}^{n}-\sum_{\beta=1}^{n-1} \sum_{\alpha=1}^{n}\right) F^{\alpha \beta} u_{\alpha \beta}+\sum_{\alpha=1}^{n} F^{j \alpha} u_{n \alpha} u_{j j}, \\
& \sum_{\alpha, \beta=1}^{n} F^{\alpha \beta} u_{j \alpha} u_{n j \beta}=u_{n j}\left(\sum_{\alpha, \beta=1}^{n}-\sum_{\alpha=1}^{n} \sum_{\beta=1}^{n-1}\right) F^{\alpha \beta} u_{\alpha \beta j}+\sum_{\alpha=1}^{n} F^{j \alpha} u_{j \alpha n} u_{j j},
\end{aligned}
$$

and by (3.20), for $j \in B$,

$$
\begin{aligned}
& u_{n} \sum_{\alpha=1}^{n} \sum_{\beta=1}^{n-1} F^{\alpha \beta} u_{\alpha \beta j}=u_{n} \sum_{\alpha=1}^{n}\left(\sum_{i \in B} F^{\alpha i} u_{i j \alpha}+\sum_{i \in G} F^{\alpha i} u_{i j \alpha}\right) \\
= & \sum_{\alpha=1}^{n} \sum_{i \in B} F^{\alpha i}\left(u_{i \alpha} u_{j n}+u_{j \alpha} u_{i n}-\eta_{0} g_{\alpha} u_{n}^{2} \delta_{i j}+u_{n \alpha} u_{i j}\right) \\
& +\sum_{\alpha=1}^{n} \sum_{i \in G} F^{\alpha i}\left(-u_{n}^{2} \widetilde{a}_{i j, \alpha}+u_{i \alpha} u_{j n}+u_{j \alpha} u_{i n}\right)+O\left(\mathcal{H}_{\phi}\right) \\
= & -u_{n}^{2} \sum_{\alpha=1}^{n} \sum_{i \in G} F^{\alpha i} \widetilde{a}_{i j, \alpha}+u_{n j} \sum_{i \in G} F^{i i} u_{i i}+2 u_{n j}\left(\sum_{i=1}^{n-1} F^{n i} u_{n i}\right)+u_{n j} \sum_{i \in B} F^{i i} u_{i i} \\
& +u_{j j} \sum_{i=1}^{n-1} F^{i j} u_{n i}+u_{j j} \sum_{i=1}^{n} F^{i j} u_{n i}-\eta_{0} \sum_{\alpha=1}^{n} F^{j \alpha} g_{\alpha} u_{n}^{2}+O\left(\mathcal{H}_{\phi}\right),
\end{aligned}
$$


and

$$
\begin{aligned}
& \sum_{\alpha, \beta=1}^{n} F^{\alpha \beta} u_{j \alpha} u_{j \beta}=F^{n n} u_{n j}^{2}+2 F^{j n} u_{j n} u_{j j}+F^{j j} u_{j j}^{2} \\
= & u_{n j}^{2}\left(\sum_{\alpha, \beta=1}^{n} F^{\alpha \beta} u_{\alpha \beta}-2 \sum_{\alpha=1}^{n-1} F^{\alpha n} u_{n \alpha}-\sum_{\alpha, \beta=1}^{n-1} F^{\alpha \beta} u_{\alpha \beta}\right)+2 F^{j n} u_{j n} u_{j j}+F^{j j} u_{j j}^{2} .
\end{aligned}
$$

Put above to (3.22),

$$
\begin{aligned}
u_{n}^{3} \sum_{\alpha, \beta=1}^{n} F^{\alpha \beta} a_{j j, \alpha \beta}= & -u_{n}^{2} \sum_{\alpha, \beta=1}^{n} F^{\alpha \beta}\left(u_{\alpha \beta j j}+6 u_{\alpha \beta j} u_{n j} u_{n}-6 u_{\alpha \beta} u_{n j}^{2}\right) \\
& +4 u_{n}^{2} u_{n j} \sum_{\alpha=1}^{n} \sum_{i \in G} F^{\alpha i} \widetilde{a}_{i j, \alpha}+2 u_{n j}^{2} \sum_{i \in G} F^{i i} u_{i i}+2 u_{n j}^{2} \sum_{i \in B} F^{i i} u_{i i} \\
& -12 \sum_{\alpha=1}^{n} F^{j \alpha} u_{n \alpha} u_{j n} u_{j j}+4 \sum_{\alpha=1}^{n} F^{j \alpha} u_{j n \alpha} u_{n} u_{j j}-2 u_{n n} F^{j j} u_{j j}^{2} \\
& -\eta_{0} g \sum_{\alpha, \beta=1}^{n} F^{\alpha \beta}\left(2 u_{j \alpha} u_{j \beta} u_{n}+u_{n \alpha \beta} u_{n}^{2}+\sum_{i=1}^{n-1} u_{i \alpha} u_{i \beta} u_{n}\right) \\
& -2 \eta_{0} \sum_{\alpha, \beta=1}^{n} F^{\alpha \beta} u_{n \alpha} g_{\beta} u_{n}+4 \eta_{0} \sum_{\alpha=1}^{n} F^{j \alpha} g_{\alpha} u_{j n} u_{n}^{2}+O\left(\mathcal{H}_{\phi}\right) .
\end{aligned}
$$

Since $a_{i j, \alpha}=\widetilde{a}_{i j, \alpha}$ for $i \neq j$,

$$
\sum_{\alpha, \beta=1}^{n} \frac{F^{\alpha \beta} \widetilde{a}_{i j, \alpha} \widetilde{a}_{i j, \beta}}{\widetilde{a}_{i i}}=\sum_{\alpha, \beta=1}^{n} \frac{F^{\alpha \beta} a_{i j, \alpha} a_{i j, \beta}}{a_{i i}} \frac{a_{i i}}{\widetilde{a}_{i i}}=\sum_{\alpha, \beta=1}^{n} \frac{F^{\alpha \beta} a_{i j, \alpha} a_{i j, \beta}}{a_{i i}}\left(1+\frac{\eta_{0} g}{\widetilde{a}_{i i}}\right) \text {. }
$$

(3.18), (3.23) and (3.24) yield that, for each $j \in B$,

$$
\begin{aligned}
F^{\alpha \beta} p_{\alpha \beta}= & F^{\alpha \beta} \sigma_{l}(G)\left[\sum_{j \in B}\left(a_{j j, \alpha \beta}-\eta_{0} g_{\alpha \beta}\right)-2 \sum_{i \in G, j \in B} \frac{\widetilde{a}_{i j, \alpha} \widetilde{a}_{i j, \beta}}{\widetilde{a}_{i i}}\right]+O\left(\mathcal{H}_{\phi}\right) \\
= & u_{n}^{-3} \sum_{j \in B} \sigma_{l}(G)\left[-\sum_{\alpha, \beta=1}^{n} F^{\alpha \beta} u_{n}^{2} u_{\alpha \beta j j}+6 u_{n} \sum_{\alpha, \beta=1}^{n} F^{\alpha \beta} u_{j n} u_{\alpha \beta j}\right. \\
& \left.-6 u_{j n}^{2} \sum_{\alpha, \beta=1}^{n} F^{\alpha \beta} u_{\alpha \beta}+I_{j}+J_{1 j}\right]+O\left(\mathcal{H}_{\phi}\right) .
\end{aligned}
$$

where $I_{j}, J_{1 j}$ as in (3.12) and (3.13).

For each $j \in B$, differentiating equation (1.1) in $e_{j}$ direction at $x$,

$$
\sum_{\alpha, \beta=1}^{n} F^{\alpha \beta} u_{\alpha \beta j}+F^{u_{n}} u_{j n}+F^{u_{j}} u_{j j}+F^{x_{j}}=0
$$




$$
\begin{aligned}
-\sum_{\alpha, \beta=1}^{n} F^{\alpha \beta} u_{\alpha \beta j j}= & \sum_{\alpha, \beta, \gamma, \eta=1}^{n} F^{\alpha \beta, \gamma \eta} u_{\alpha \beta j} u_{\gamma \eta j}+2 \sum_{\alpha, \beta, l=1}^{n} F^{\alpha \beta, u_{l}} u_{\alpha \beta j} u_{l j} \\
& +2 \sum_{\alpha, \beta=1}^{n} F^{\alpha \beta, x_{j}} u_{\alpha \beta j}+\sum_{l, s=1}^{n} F^{u_{l}, u_{s}} u_{l j} u_{s j}+F^{u} u_{j j} \\
& +\sum_{l=1}^{n} F^{u_{l}, x_{j}} u_{l j}+F^{x_{j}, x_{j}}+\sum_{l=1}^{n} F^{u_{l}} u_{l j j} .
\end{aligned}
$$

It follows from (3.20) that, at $x$

$$
\begin{aligned}
-\sum_{\alpha, \beta=1}^{n} F^{\alpha \beta} u_{\alpha \beta j j}= & \sum_{\alpha, \beta, \gamma, \eta=1}^{n} F^{\alpha \beta, \gamma \eta} u_{\alpha \beta j} u_{\gamma \eta j}+2 \sum_{\alpha, \beta=1}^{n}\left[F^{\alpha \beta, u_{n}} u_{j \alpha \beta} u_{n j}+F^{\alpha \beta, x_{j}} u_{\alpha \beta j}\right] \\
& +F^{u_{n}, u_{n}} u_{j n}^{2}+2 F^{u_{n}, x_{j}} u_{j n}+F^{x_{j}, x_{j}}+2 \frac{F^{u_{n}}}{u_{n}} u_{j n}^{2}+J_{2 j}+O\left(\mathcal{H}_{\phi}\right),
\end{aligned}
$$

where $J_{2 j}$ is defined in (3.14).

Since $u_{\alpha \beta j j}=u_{j j \alpha \beta}$, from (3.25) and (3.28),

$$
\begin{aligned}
F^{\alpha \beta} p_{\alpha \beta}= & \sum_{j \in B} u_{n}^{-3} \sigma_{l}(G)\left[\left(\sum_{\alpha, \beta, \gamma, \eta=1}^{n} F^{\alpha \beta, \gamma \eta} u_{\alpha \beta j} u_{\gamma \eta j}+2 \sum_{\alpha, \beta=1}^{n} F^{\alpha \beta, u_{n}} u_{j \alpha \beta} u_{j n}\right.\right. \\
& \left.+2 \sum_{\alpha, \beta=1}^{n} F^{\alpha \beta, x_{j}} u_{j \alpha \beta}+F^{u_{n}, u_{n}} u_{j n}^{2}+2 F^{u_{n}, x_{j}} u_{j n}+F^{x_{j}, x_{j}}+2 \frac{F^{u_{n}}}{u_{n}} u_{j n}^{2}\right) u_{n}^{2} \\
& \left.+6 \sum_{\alpha, \beta=1}^{n} F^{\alpha \beta} u_{j \alpha \beta} u_{j n} u_{n}-6 \sum_{\alpha, \beta=1}^{n} F^{\alpha \beta} u_{\alpha \beta} u_{j n}^{2}+I_{j}+J_{1 j}+J_{2 j}\right]+O\left(\mathcal{H}_{\phi}\right) .
\end{aligned}
$$

The fact $q \in C^{1,1}(\mathcal{O})$ follows Corollary 2.2 in [1]. Also by Lemma 2.4 in [1],

$$
q_{\alpha}=\frac{\partial q}{\partial x_{\alpha}}=\sum_{j \in B} \frac{\sigma_{1}^{2}(B \mid j)-\sigma_{2}(B \mid j)}{\sigma_{1}^{2}(B)} \widetilde{a}_{j j, \alpha}+O\left(\mathcal{H}_{\phi}\right),
$$

and

$$
\begin{aligned}
q_{\alpha \beta}= & \sum_{j \in B} \frac{\sigma_{1}^{2}(B \mid j)-\sigma_{2}(B \mid j)}{\sigma_{1}^{2}(B)}\left[\widetilde{a}_{j j, \alpha \beta}-2 \sum_{i \in G} \frac{\widetilde{a}_{i j, \alpha} \widetilde{a}_{i j, \beta}}{\widetilde{a}_{i i}}\right] \\
& -\frac{1}{\sigma_{1}^{3}(B)} \sum_{i \in B}\left[\sigma_{1}(B) \widetilde{a}_{i i, \alpha}-\widetilde{a}_{i i} \sum_{j \in B} \widetilde{a}_{j j, \alpha}\right]\left[\sigma_{1}(B) \widetilde{a}_{i i, \beta}-\widetilde{a}_{i i} \sum_{j \in B} \widetilde{a}_{j j, \beta}\right] \\
& -\frac{1}{\sigma_{1}(B)} \sum_{i \neq j \in B} \widetilde{a}_{i j, \alpha} \widetilde{a}_{i j, \beta}+O\left(\mathcal{H}_{\phi}\right) .
\end{aligned}
$$


Following the same computations as for $p$, we get

$$
\begin{aligned}
& \sum_{\alpha, \beta=1}^{n} F^{\alpha \beta} q_{\alpha \beta} \\
= & \sum_{j \in B} \frac{\sigma_{1}^{2}(B \mid j)-\sigma_{2}(B \mid j)}{\sigma_{1}^{2}(B) u_{n}^{3}}\left[\left(\sum_{\alpha, \beta, \gamma, \eta=1}^{n} F^{\alpha \beta, \gamma \eta} u_{\alpha \beta j} u_{\gamma \eta j}+2 \sum_{\alpha, \beta=1}^{n} F^{\alpha \beta, u_{n}} u_{j \alpha \beta} u_{j n}\right.\right. \\
& \left.+2 \sum_{\alpha, \beta=1}^{n} F^{\alpha \beta, x_{j}} u_{j \alpha \beta}+F^{u_{n}, u_{n}} u_{j n}^{2}+2 F^{u_{n}, x_{j}} u_{j n}+F^{x_{j}, x_{j}}+2 \frac{F^{u_{n}}}{u_{n}} u_{j n}^{2}\right) u_{n}^{2} \\
& \left.+6 \sum_{\alpha, \beta=1}^{n} F^{\alpha \beta} u_{j \alpha \beta} u_{j n} u_{n}-6 \sum_{\alpha, \beta=1}^{n} F^{\alpha \beta} u_{\alpha \beta} u_{j n}^{2}+I_{j}+J_{1 j}+J_{2 j}\right] \\
& -\frac{1}{\sigma_{1}^{3}(B)} \sum_{\alpha, \beta=1}^{n} \sum_{i \in B} F^{\alpha \beta}\left[\sigma_{1}(B) \widetilde{a}_{i i, \alpha}-\widetilde{a}_{i i} \sum_{j \in B} \widetilde{a}_{j j, \alpha}\right]\left[\sigma_{1}(B) \widetilde{a}_{i i, \beta}-\widetilde{a}_{i i} \sum_{j \in B} \widetilde{a}_{j j, \beta}\right] \\
& -\frac{1}{\sigma_{1}(B)} \sum_{\alpha, \beta=1}^{n} \sum_{i \neq j \in B} F^{\alpha \beta} \widetilde{a}_{i j, \alpha} \widetilde{a}_{i j, \beta}+O\left(\mathcal{H}_{\phi}\right) .
\end{aligned}
$$

The proof of the Lemma is complete.

\section{Proof of Theorems}

We want to show $\widetilde{a}$ defined in (3.4) is of constant rank. Theorem 1.3 corresponds to the case $\eta_{0}=0$. As for Theorem 1.4, set

$$
\Omega_{\varpi}=\left\{x \in \Omega \mid 0<\kappa_{s}(x)<\frac{\lambda}{100 \varpi} \cdot\right\}
$$

We indicate how the constant rank theorem for $\widetilde{a}$ would imply Theorem 1.4. If $\min \left\{\kappa^{0}, \kappa^{1}\right\}=$ 0 , the strict convexity of level surfaces $\Sigma^{c}$ for $c \in(0,1)$ in Theorem 1.4 follows from Theorem 1.3. We may assume $\min \left\{\kappa^{0}, \kappa^{1}\right\}>0$. By Theorem 1.4, $a$ is strictly positive definite in $\bar{\Omega}$. That is, $\kappa_{s}(x)>0, \forall x \in \bar{\Omega}$. By the continuity, $\kappa_{s}(x)$ has a positive lower bound (which we want to estimate). Increasing $\eta_{0}$ from 0 to the level that $\widetilde{a}$ is nonnegative definite through out $\bar{\Omega}_{\varpi}$ but degenerate at some points $x_{0}$. (1.12) follows easily if the degeneracy happens outside $\Omega_{\varpi}$ or on the boundary. If the degeneracy happens at an interior point $x_{0}$ of $\Omega_{\varpi}$, the goal is to show that $\widetilde{a}$ is degenerate through out the connected component $U$ of $\Omega_{\varpi}$ containing $x_{0}$ with the same rank. If this is true, $\kappa_{s}(x)=$ constant, $\forall x \in \Sigma^{c} \bigcap U$. Theorem 1.4 would follow. Furthermore, the closure of $\Sigma^{u(x)} \bigcap U$ can not intersect the set $\left\{z \in \Omega \mid \kappa_{s}(z)=\frac{\lambda}{100 \varpi}\right\}$ for any $x \in U$, since by definition $\kappa_{s}(x)<\frac{\lambda}{100 \varpi}$. This would imply that if the degeneracy of $\widetilde{a}$ happens at an interior point of $\Omega_{\varpi}$, the connect component $U$ of $\Omega_{\varpi}$ containing that point is exactly the set $\bigcup_{c_{0}<c<c_{1}} \Sigma^{c}$ for some $0 \leqslant c_{0}<c_{1} \leqslant 1$, and $\kappa_{s}(x) \equiv$ constant, $\forall x \in \Sigma^{c}$. Therefore, $\Sigma^{c}$ is a round sphere for each $c_{0} \leqslant c \leqslant c_{1}$. The task now is to prove the rank of $\widetilde{a}$ is constant. That is a consequence of the following proposition. 
Proposition 4.1. Suppose $u \in C^{3,1}$ is a quasiconcave solution of equation (1.1) and $F$ satisfies assumptions in Theorem 1.3. If the second fundamental form of $\Sigma^{c}$ of solution $u$ attains minimum rank $l$ at certain point $x_{0} \in \Omega$, then there exist a neighborhood $\mathcal{O}$ of $x_{0}$ and a positive constant $C$ independent of $\phi$ (defined in (3.8) ), such that

$$
\sum_{\alpha, \beta=1}^{n} F^{\alpha \beta} \phi_{\alpha \beta}(x) \leqslant C(\phi(x)+|\nabla \phi(x)|), \forall x \in \mathcal{O} .
$$

If in addition $F$ satisfies the uniform ellipticity condition (1.10) in $\mathcal{O} \subset \Omega_{\varpi}$, then there is $A$ depending only on $\|F\|_{C^{2}}, n, \lambda, d_{0},\|u\|_{C^{4}(\bar{\Omega})}$, such that if $\widetilde{a}(x)$ defined in (3.4) attains minimum rank $l$ at certain point $x_{0} \in \mathcal{O}$, then inequality (4.2) is true for all $x \in \mathcal{O}$.

Proof of Proposition 4.1. Suppose the minimum rank $l$ of $\widetilde{a}$ is attained at an interior point $x_{0}$, and we may assume $l \leqslant n-2$. Let $\mathcal{O}$ be a small neighborhood of $x_{0}$. Lemma 3.1 and (3.8) implies $\phi \in C^{1,1}(\mathcal{O}), \phi(x) \geqslant 0, \phi\left(x_{0}\right)=0$. For $\epsilon>0$ sufficient small, let $\phi_{\epsilon}$ defined as in (3.9), we want to establish differential inequality (4.2) for $\phi_{\varepsilon}$ with constant $C$ independent of $\varepsilon$ in $\mathcal{O}$. For each fixed $x \in \mathcal{O}$, choose a local coordinate frame $e_{1}, \cdots, e_{n-1}, e_{n}$ so (3.5) and (3.6) are satisfied. We will omit the subindex $\varepsilon$ with the understanding that all the estimates are independent of $\varepsilon$. From Lemma 3.1 ,

$$
\begin{aligned}
& F^{\alpha \beta} \phi_{\alpha \beta} \\
= & \sum_{j \in B} u_{n}^{-3}\left[\sigma_{l}(G)+\frac{\sigma_{1}^{2}(B \mid j)-\sigma_{2}(B \mid j)}{\sigma_{1}^{2}(B)}\right]\left\{S_{j}+I_{j}+J_{1 j}+J_{2 j}\right\} \\
& -\frac{1}{\sigma_{1}^{3}(B)} \sum_{\alpha, \beta=1}^{n} \sum_{i \in B} F^{\alpha \beta}\left[\sigma_{1}(B) \widetilde{a}_{i i, \alpha}-\widetilde{a}_{i i} \sum_{j \in B} \widetilde{a}_{j j, \alpha}\right]\left[\sigma_{1}(B) \widetilde{a}_{i i, \beta}-\widetilde{a}_{i i} \sum_{j \in B} \widetilde{a}_{j j, \beta}\right] \\
& -\frac{1}{\sigma_{1}(B)} \sum_{\alpha, \beta=1}^{n} \sum_{i \neq j, i, j \in B} F^{\alpha \beta} \widetilde{a}_{i j, \alpha} \widetilde{a}_{i j, \beta}+O\left(\mathcal{H}_{\phi}\right),
\end{aligned}
$$

where $I_{j}, J_{1 j}, J_{2 j}$ are defined in (3.12), (3.13), (3.14) respectively, and,

$$
\begin{aligned}
S_{j}= & {\left[\sum_{\alpha, \beta, \gamma, \eta=1}^{n} F^{\alpha \beta, \gamma \eta} u_{j \alpha \beta} u_{\gamma \eta j}+2 \sum_{\alpha, \beta=1}^{n} F^{\alpha \beta, u_{n}} u_{j \alpha \beta} u_{j n}+2 \sum_{\alpha, \beta=1}^{n} F^{\alpha \beta, x_{j}} u_{j \alpha \beta}\right.} \\
+ & \left.F^{u_{n}, u_{n}} u_{j n}^{2}+2 F^{u_{n}, x_{j}} u_{j n}+F^{x_{j}, x_{j}}+2 \frac{F^{u_{n}}}{u_{n}} u_{j n}^{2}\right] u_{n}^{2} \\
+ & 6 \sum_{\alpha, \beta=1}^{n} F^{\alpha \beta} u_{j \alpha \beta} u_{j n} u_{n}-6 \sum_{\alpha, \beta=1}^{n} F^{\alpha \beta} u_{\alpha \beta} u_{j n}^{2}
\end{aligned}
$$

In the coordinate system (3.5),

$$
t=u_{n}^{-1}, \quad D^{2} u(x)=t^{-1} \widetilde{\mathbf{A}}, \quad A_{i j}=t u_{i j}=\frac{u_{i j}}{u_{n}}, \quad \theta=(0, . ., 0,1) .
$$


For each $j \in B$, set

$$
\begin{aligned}
\tilde{X}_{\alpha \beta}= & u_{\alpha \beta j} u_{n} ; \quad \forall \alpha, \beta \in G \cup\{n\} \quad \text { with } \quad(\alpha, \beta) \neq(n, n), \\
\widetilde{X}_{\alpha \beta}= & 2 u_{\alpha \beta} u_{j n} ; \quad \forall \alpha \in B \text { or } \forall \beta \in B, \\
\widetilde{X}_{n n}= & u_{n n j} u_{n}+\frac{1}{F^{n n}}\left[2 \sum_{\substack{\alpha \in B \\
\beta \in G \cup\{n\}}} F^{\alpha \beta} u_{\alpha \beta j} u_{n}+\sum_{\alpha, \beta \in B} F^{\alpha \beta} u_{\alpha \beta j} u_{n}\right. \\
& \left.\quad-2 \sum_{\alpha, \beta \in B} F^{\alpha \beta} u_{\alpha \beta} u_{j n}-4 \sum_{\alpha \in B} F^{\alpha n} u_{\alpha n} u_{j n}+F^{u_{j}} u_{j j} u_{n}\right] ; \\
\widetilde{Y}= & u_{j n} u_{n} ; \quad Z_{k}=\delta_{k j} u_{n} .
\end{aligned}
$$

For such $\widetilde{V}=\left(\left(\tilde{X}_{\alpha \beta}\right), \tilde{Y},\left(Z_{k}\right)\right)$, by (3.26),

$$
\begin{aligned}
<V, \nabla_{(B, x)} F> & =F^{\alpha \beta} \widetilde{X}_{\alpha \beta}+F^{u_{n}} \tilde{Y}+F^{x_{k}} Z_{k} \\
& =F^{\alpha \beta} u_{\alpha \beta j} u_{n}+F^{u_{j}} u_{j j} u_{n}+F^{u_{n}} u_{j n} u_{n}+F_{x_{j}} u_{n}=0 .
\end{aligned}
$$

We need the following lemma.

Lemma 4.2. Under the coordinate system (3.5) at $x$ with $\widetilde{V}$ as in (4.6), then

$$
u_{n}^{3} I=I_{j}+O\left(u_{j j}\right),
$$

where $I_{j}$ defined in (3.12) and $I$ defined in (2.26).

Proof of Lemma 4.2. Since $u_{i i}=-a_{i i} u_{n}$ for $i \in G$ and $\left(u_{i j}\right), i, j=1, . ., n-1$ is diagonal at $x$. By (3.12) and (3.20), for each $j \in B$,

$$
\begin{aligned}
I_{j} & =\sum_{i \in G}\left[-2 u_{n}^{3} \sum_{\alpha, \beta \notin B} \frac{F^{\alpha \beta} a_{i j, \alpha} a_{i j, \beta}}{a_{i i}}+4 u_{n}^{2} u_{n j} \sum_{\alpha \notin B} F^{\alpha i} a_{i j, \alpha}+2 u_{n j}^{2} F^{i i} u_{i i}\right] \\
& =\sum_{i \in G} \sum_{\alpha, \beta \notin B}\left[2 u_{n}^{2} \frac{F^{\alpha \beta} u_{i j, \alpha} u_{i j, \beta}}{u_{i i}}-8 u_{n} u_{n j} \frac{F^{\alpha \beta} u_{i j, \alpha} u_{i \beta}}{u_{i i}}+8 u_{n j}^{2} \frac{F^{\alpha \beta} u_{i \alpha} u_{i \beta}}{u_{i i}}\right]+O\left(u_{j j}\right) \\
(4.7) & =2 \sum_{i \in G} \sum_{\alpha, \beta \notin B} \frac{F^{\alpha \beta}\left(u_{i j \alpha} u_{n}-2 u_{i \alpha} u_{j n}\right)\left(u_{i j \beta} u_{n}-2 u_{i \beta} u_{j n}\right)}{u_{i i}}+O\left(u_{j j}\right) .
\end{aligned}
$$

By (4.5), (2.23), (2.25) and (4.6),

$$
Y_{i \alpha}=0, \forall i \in B ; \quad Y_{i \alpha}=-\left(u_{i j \alpha} u_{n}^{-1}-2 u_{i \alpha} u_{j n} u_{n}^{-2}\right), \forall i \in G, \alpha \notin B .
$$

Therefore

$$
u_{n}^{3} I=2 \sum_{i \in G} \sum_{\alpha, \beta \notin B} \frac{F^{\alpha \beta}}{u_{i i}} Y_{i \alpha} Y_{i \beta} u_{n}^{4}=I_{j}+O\left(u_{j j}\right) .
$$

We now finish the proof of the proposition. 
In case of Theorem 1.3, since $\eta_{0}=0, u_{j j}=O(\phi), \forall j \in B, a=\widetilde{a}$. (4.3) gives

$$
\begin{aligned}
& F^{\alpha \beta} \phi_{\alpha \beta} \\
= & \sum_{j \in B} u_{n}^{-3}\left[\sigma_{l}(G)+\frac{\sigma_{1}^{2}(B \mid j)-\sigma_{2}(B \mid j)}{\sigma_{1}^{2}(B)}\right]\left(S_{j}+I_{j}\right) \\
& -\frac{1}{\sigma_{1}^{3}(B)} \sum_{\alpha, \beta=1}^{n} \sum_{i \in B} F^{\alpha \beta}\left[\sigma_{1}(B) a_{i i, \alpha}-a_{i i} \sum_{j \in B} a_{j j, \alpha}\right]\left[\sigma_{1}(B) a_{i i, \beta}-a_{i i} \sum_{j \in B} a_{j j, \beta}\right] \\
& -\frac{1}{\sigma_{1}(B)} \sum_{\alpha, \beta=1}^{n} \sum_{i \neq j, i, j \in B} F^{\alpha \beta} a_{i j, \alpha} a_{i j, \beta}+O\left(\mathcal{H}_{\phi}\right) .
\end{aligned}
$$

In fact for each $j \in B$, by (4.6) and (3.20),

$$
\begin{aligned}
& \widetilde{X}_{\alpha \beta}=u_{\alpha \beta j} u_{n}+O\left(\mathcal{H}_{\phi}\right) ; \quad \forall \alpha \in B \text { or } \forall \beta \in B, \\
& \widetilde{X}_{\alpha \beta}=u_{\alpha \beta j} u_{n} ; \quad \forall \alpha, \beta \in G \cup\{n\},(\alpha, \beta) \neq(n, n), \\
& \widetilde{X}_{n n}=u_{n n j} u_{n}+O\left(\mathcal{H}_{\phi}\right) .
\end{aligned}
$$

Lemma 4.2, condition (1.8) and Lemma 2.6 imply

$$
S_{j}+I_{j}=H(\tilde{V}, \tilde{V})+O\left(\mathcal{H}_{\phi}\right) \leqslant O\left(\mathcal{H}_{\phi}\right) .
$$

By the Newton-MacLaurine inequality, $C \geqslant \sigma_{l}(G)+\frac{\sigma_{1}^{2}(B \mid j)-\sigma_{2}(B \mid j)}{\sigma_{1}^{2}(B)} \geqslant 0$. Condition (1.7) implies that there is $\delta>0$

$$
\left(F^{\alpha \beta}\right) \geqslant \delta I, \forall x \in \mathcal{O} .
$$

Combining (4.9), (4.11) and (4.12)

$$
\begin{aligned}
F^{\alpha \beta} \phi_{\alpha \beta} \leqslant \quad & C\left(\phi+\sum_{i, j \in B}\left|\nabla a_{i j}\right|\right)-\frac{\delta}{\sigma_{1}(B)} \sum_{\alpha=1}^{n} \sum_{i \neq j \in B} a_{i j \alpha}^{2} \\
& -\frac{\delta}{\sigma_{1}^{3}(B)} \sum_{\alpha=1}^{n} \sum_{i \in B}\left(\sigma_{1}(B) a_{i i, \alpha}-a_{i i} \sum_{j \in B} a_{j j, \alpha}\right)^{2} .
\end{aligned}
$$

Finally, by Lemma 3.3 in [1], the term $\sum_{i, j \in B}\left|\nabla a_{i j}\right|$ can be controlled by the rest terms on the right hand side in (4.13) and $\phi+|\nabla \phi|$. In conclusion, there exist positive constant $C$ independent of $\varepsilon$, such that

$$
\sum_{\alpha, \beta} F^{\alpha \beta} \phi_{\alpha \beta} \leqslant C(\phi+|\nabla \phi|) .
$$

Proposition 4.1 is verified under the assumptions of Theorem 1.3. Therefore, Theorem 1.3 is proved.

The rest proof is to deal with the case $\eta_{0}>0$. In this case, since $\kappa_{s}(x)>0$ for all $x \in \Omega$ by Theorem 1.3 , Since $\mathcal{O} \subset \Omega_{\varpi}$,

$$
0<\kappa_{s}(x)<\frac{\lambda}{100 \varpi}, \forall x \in \mathcal{O}
$$


For each $j \in B$, it follows from (4.6), (3.20), (3.21), and the fact $u_{k}=0$ for all $k \leqslant n-1$,

$$
\begin{aligned}
\widetilde{X}_{\alpha \beta} & =u_{\alpha \beta j} u_{n}+\widetilde{a}_{\alpha j \beta} u_{n}^{2}+\eta_{0} g_{n} u_{n}^{2} \delta_{\beta n} \delta_{\alpha j}+O\left(u_{j j}\right), \quad \forall \alpha \in B ; \\
\widetilde{X}_{\alpha \beta} & =u_{\alpha \beta j} u_{n} ; \forall \alpha, \beta \in G \cup\{n\},(\alpha, \beta) \neq(n, n), \\
\widetilde{X}_{n n} & =u_{n n j} u_{n}-\frac{2 \eta_{0} g_{n} u_{n}^{2} F^{n j}}{F^{n n}}+O\left(\sum_{i, j \in B}\left|\nabla \widetilde{a}_{i j}\right|\right)+O\left(u_{j j}\right) .
\end{aligned}
$$

In view of (4.16),

$$
\begin{aligned}
& u_{\alpha \beta j} u_{n}=\widetilde{X}_{\alpha \beta}-\widetilde{a}_{\alpha j \beta} u_{n}^{2}-\eta_{0} g_{n} u_{n}^{2} \delta_{\beta n} \delta_{\alpha j}+O\left(u_{j j}\right), \quad \forall \alpha \in B ; \\
& u_{\alpha \beta j} u_{n}=\widetilde{X}_{\alpha \beta} ; \forall \alpha, \beta \in G \cup\{n\},(\alpha, \beta) \neq(n, n), \\
& u_{n n j} u_{n}=\widetilde{X}_{n n}+\frac{2 \eta_{0} g_{n} u_{n}^{2} F^{n j}}{F^{n n}}+O\left(\sum_{i, j \in B}\left|\nabla \widetilde{a}_{i j}\right|\right)+O\left(u_{j j}\right) .
\end{aligned}
$$

Notice that $u_{j j}=-\eta_{0} g u_{n}+O(\phi), \forall j \in B$. Substitute $u_{\alpha \beta j} u_{n}$ by formula (4.17) in $S_{j}$ defined in (4.4). We need to track the terms with factor $\eta_{0}^{2} g_{n}^{2}$. They are coming from $\sum_{\alpha, \beta, \gamma, \eta \in\{j, n\}} F^{\alpha \beta, \gamma \eta} u_{j \alpha \beta} u_{\gamma \eta j} u_{n}^{2}$ only. In turn, the coefficient in front of $\eta_{0}^{2} g_{n}^{2}$ can be controlled by, say $50 \varpi \eta_{0}^{2} u_{n}^{3}$, where $\varpi$ is defined in (1.11). By Lemma 4.2, condition (1.8), Lemma 2.6 and the assumptions of Theorem 1.4 there exist constants $C_{0}^{\prime}, C_{0}^{\prime \prime}$ depending only on $\lambda,\|F\|_{C^{2}}, d_{0},\|u\|_{C^{3}}$ such that

$$
\begin{aligned}
S_{j}+I_{j} & \leqslant H(\widetilde{V}, \widetilde{V})+50 \varpi \eta_{0}^{2} g_{n}^{2} u_{n}^{3}+C_{0}^{\prime} \eta_{0}\left|g_{n}\right|+C_{0}^{\prime \prime} \eta_{0} g+O\left(\mathcal{H}_{\phi}\right) \\
& \leqslant 50 \varpi \eta_{0}^{2} g_{n}^{2} u_{n}^{3}+C_{0}^{\prime} \eta_{0}\left|g_{n}\right|+C_{0}^{\prime \prime} \eta_{0} g+O\left(\mathcal{H}_{\phi}\right) \\
& =\left(50 \varpi \eta_{0} g\right) A^{2} \eta_{0} g u_{n}^{5}+C_{0}^{\prime} A \eta_{0} g u_{n}+C_{0}^{\prime \prime} \eta_{0} g+O\left(\mathcal{H}_{\phi}\right) .
\end{aligned}
$$

Since $F^{n n} \geqslant \lambda$, by (3.13) and (3.14),

$$
\begin{aligned}
J_{1 j}+J_{2 j}+O\left(u_{j j}\right) & \leqslant-\eta_{0} F^{n n} g_{n n} u_{n}^{3}+\eta_{0} C_{1}^{\prime}\left|g_{n}\right|+\eta_{0} C_{2}^{\prime} g+O(\phi) \\
& \leqslant-\eta_{0} g \lambda A^{2} u_{n}^{5}+\eta_{0} g A C_{1}^{\prime \prime} u_{n}+\eta_{0} C_{2}^{\prime} g+O(\phi),
\end{aligned}
$$

where $C_{1}^{\prime}, C_{1}^{\prime \prime} C_{2}^{\prime}$ are positive constants depending only on $n, d_{0},\|u\|_{C^{3}(\Omega)},\|F\|_{C^{2}}$. Note that $\eta_{0} g \leqslant \kappa_{s}(x)<\frac{\lambda}{100 \varpi}$, it follows from (4.15) that

$$
S_{j}+I_{j}+J_{1 j}+J_{2 j}+O\left(u_{j j}\right) \leqslant \frac{\eta_{0} g}{2}\left(-A^{2} \lambda u_{n}^{5}+C_{3}^{\prime} A+C_{3}^{\prime \prime}\right)+O\left(\mathcal{H}_{\phi}\right) .
$$

where $C_{3}^{\prime}, C_{3}^{\prime \prime}$ are positive constants depending only on $n, d_{0},\|u\|_{C^{3}(\Omega)},\|F\|_{C^{2}}$. Since $u_{n} \geqslant$ $d_{0}>0$, we may choose $A$ large enough in (4.20) depending only on $n, d_{0},\|u\|_{C^{3}(\Omega)},\|F\|_{C^{2}}, \lambda$ such that

$$
S_{j}+I_{j}+J_{1 j}+J_{2 j}+O\left(u_{j j}\right) \leqslant O\left(\mathcal{H}_{\phi}\right)
$$

By (4.3) and (4.21),

$$
\begin{aligned}
F^{\alpha \beta} \phi_{\alpha \beta} \leqslant \quad & C\left(\phi+\sum_{i, j \in B}\left|\nabla \tilde{a}_{i j}\right|\right)-\frac{\delta}{\sigma_{1}(B)} \sum_{\alpha=1}^{n} \sum_{i \neq j \in B} \tilde{a}_{i j \alpha}^{2} \\
& -\frac{\delta}{\sigma_{1}^{3}(B)} \sum_{\alpha=1}^{n} \sum_{i \in B}\left(\sigma_{1}(B) \tilde{a}_{i i, \alpha}-\tilde{a}_{i i} \sum_{j \in B} \tilde{a}_{j j, \alpha}\right)^{2} .
\end{aligned}
$$

As in the case of $\eta_{0}=0$, the same argument yields (4.14) for $\tilde{a}$. Thus Proposition 4.1 is validated under the assumptions in Theorem 1.4 
We remark that Theorem 1.4 covers all quasilinear equations satisfying structural conditions (1.8)-(1.10). Therefore, it covers the quasilinear equations treated in [11, 14] from the discussion in Section 2. In particular, $\varpi \equiv 0$ if $F$ is quasilinear. In this case, (1.12) becomes

$$
\kappa^{c} \geqslant \min \left\{\kappa^{0} e^{A c} ; \kappa^{1} e^{A(c-1)}\right\}, \quad \forall c \in[0,1]
$$

From the proof above, the strong maximum principle concludes that if " = "holds for some $c_{0} \in(0,1)$ in (4.23), then $\kappa_{s}(x) \equiv$ constant for all $x \in \Sigma^{c}$ and $\forall c \in(0,1)$. This implies that $\Sigma^{u(x)}$ is a round sphere for every $x \in \bar{\Omega}$. The same conclusion is also true if condition (1.6) is held. Note that $\varpi$ was used only in (4.18) to get (4.20). It's proved in [2] that $S_{j} \leqslant 0$ under condition (1.6). In that case, one may take $\varpi=0$ in (4.18).

Acknowledgment: We would like to thank Yong Huang for many helpful discussions. Part of the work was done while the second named author was visiting the McGill University in 2009, she would like to thank the institution for the warm hospitality.

\section{REFERENCES}

1. B. Bian and P. Guan, A microscopic convexity principle for nonlinear partial differential equations, Inventiones Math., 177, (2009), 307-335.

2. B. Bian, P. Guan, X. Ma and L. Xu, A constant rank theorem for quasiconcave solutions of fully nonlinear partial differential equations, to appear in Indiana Univ. Math. J..

3. C. Bianchini, M. Longinetti and P. Salani, Quasiconcave solutions to elliptic problems in convex rings, Indiana Univ. Math. J. 58 (2009), 1565-1590.

4. L. Caffarelli, L. Nirenberg and J. Spruck, Nonlinear second order elliptic equations IV: Starshaped compact Weingarten hypersurfaces, Current topics in partial differential equations, Y.Ohya, K.Kasahara and N.Shimakura (eds), Kinokunize, Tokyo, 1985, 1-26.

5. L. Caffarelli and J. Spruck, Convexity properties of solutions to some classical variational problems, Comm. Part. Diff. Eq. 7 (1982), 1337-1379.

6. A. Colesanti and P. Salani, Quasi-concave envelope of a function and convexity of level sets of solutions to elliptic equations, Math. Nachr. 258 (2003), 3-15.

7. P. Cuoghi and P. Salani, Convexity of level sets for solutions to nonlinear elliptic problems in convex rings, Electronic J. Diff. Eq. 2006(124) (2006), 1-12.

8. R. Gabriel, A result concerning convex level surfaces of 3-dimensional harmonic functions, J. London Math. Soc. 32 (1957), 286-294.

9. A. Greco, Quasi-concavity for semilinear elliptic equations with non-monotone and anisotrpic nonlinearities, Boundary Value Problems (2006), article ID80347, 1-15.

10. B. Kawhol, Rearrangements and convexity of level sets in PDE, Springer Lecture Notes in Math. 1150 (1985).

11. N. Korevaar, Convexity of level sets for solutions to elliptic ring problems, Comm. Part. Diff. Eq. 15(4) (1990), 541-556.

12. J. Lewis, Capacitary functions in convex rings, Arch. Rat. Mech. Anal. 66 (1977), 201-224.

13. M. Longinetti and P. Salani, On the Hessian matrix and Minkowski addition of quasiconvex functions. J. Math. Pures Appl. 88, (2007), 276-292.

14. L. Xu, A Microscopic convexity theorem of level sets for solutions to elliptic equations, Calculus of Variations and PDEs, DOI: 10.1007/s00526-010-0333-3. 
Department of Mathematics, McGill University, Montreal, Quebec. H3A 2K6, Canada.

E-mail address: guan@math.mcgill.ca

Wuhan Institute of Physics and Mathematics, The Chinese Academy of Science, Wuhan,430071, Hubei Province, China

E-mail address: xulu@wipm.ac.cn 